\title{
Study of Geometric Porosity on Static Stability and Drag using Computational Fluid Dynamics for Rigid Parachute Shapes
}

\author{
James S. Greathouse* and Alan M. Schwing ${ }^{\dagger}$ \\ Applied Aeroscience and CFD Branch \\ NASA Johnson Space Center, Houston, TX, 77584
}

\begin{abstract}
This paper explores use of computational fluid dynamics to study the effect of geometric porosity on static stability and drag for NASA's Multi-Purpose Crew Vehicle main parachute. Both of these aerodynamic characteristics are of interest to in parachute design, and computational methods promise designers the ability to perform detailed parametric studies and other design iterations with a level of control previously unobtainable using ground or flight testing. The approach presented here uses a canopy structural analysis code to define the inflated parachute shapes on which structured computational grids are generated. These grids are used by the computational fluid dynamics code OVERFLOW and are modeled as rigid, impermeable bodies for this analysis. Comparisons to Apollo drop test data is shown as preliminary validation of the technique. Results include several parametric sweeps through design variables in order to better understand the trade between static stability and drag. Finally, designs that maximize static stability with a minimal loss in drag are suggested for further study in subscale ground and flight testing.
\end{abstract}

\section{Introduction}

Flight testing of the MPCV parachute system in 2-main cluster configurations (representing a failure of one main parachute), has indicated a possibility of a periodic swinging of the MPCV in the "weak axis (defined by a line between the centers of the 2 chutes). This periodic motion is described as "pendulum" and has been observed in several flights. ${ }^{1}$ The amplitude of this swing can lead to touchdown velocities and angles that are outside of the allowable envelope. A multi-faceted approach to mitigate this issue was undertaken, with one facet involving studying improvements to the MPCV main parachute aerodynamic stability with a goal to eliminate, or reduce the magnitude of the sustained periodic swinging motion. Implicit in this effort is the assumption that the parachute aerodynamic stability is a cause, or at least a contributor, to the pendulous motion. Since flight and ground testing of design options is expensive, time consuming, and can have challenges in control of test conditions, an analysis capability that can predict canopy aerodynamic trends at relatively low cost was desired for parametric analysis of canopy modifications. Ideally, the analysis capability would be used to perform trade studies and identify a few promising parachute modifications that would then be validated by subscale wind tunnel and flight testing before proceeding to the full-scale flight testing.

One option considered was the use of computational tools that modeled both the aerodynamics and the canopy structure (fluid-structure-interaction modeling), but it was decided this method would require more time and cost to analyze the matrix of desired configurations than was available to the team. In order to fit within the available schedule available, the authors believed that a only a subset of the actual physics could be modeled, with the knowledge that reducing the fidelity of the analysis would allow only relative performance and trends between parachute configurations and accurate absolute aerodynamic performance would not be

\footnotetext{
*Engineer.

$\dagger$ Engineer, AIAA Member.
} 
predicted by this tool. Previous experience with the OVERFLOW flow solver on complex geometries lead the team to believe that multiple geometric configurations and flow attitudes could be computed relatively quickly, with the assumptions of rigid, non-permeable, non-moving geometry.

In reducing the modeled physics to this extent, for the trends to be useful we require the static stability of a single canopy to be a contributor, or at least an indicator, of the overall system stability. Also, since the selected method does not capture parachute motion (neither material deformation/flex or bulk motion such as pitching) or aerodynamic damping, material permeability, nor chute-to-to parachute aerodynamic interaction effects so these effects must be consistent between all shapes or secondary to the overall system stability for this analysis to give meaningful results.

\section{Chute Geometry Generation}

The MPCV main canopy is sized to achieve a decent rate of no more than 33 feet-per-second in a 2chute cluster (nominal operations utilize 3 parachutes for redundancy). Space and weight constraints on the parachute system led to the selection of a 116' diameter quarter-spherical ring-sail configuration with a relatively high drag coefficient achieved partly by low overall porosity.

Ring-sail canopies typically have the first several material panels near the center of the parachute built in such a way that they will inflate to the intended shape (quarter spherical in the case of the MPCV main canopy) with no additional slack - or "fullness". The remaining material panels progressing from the center toward the skirt contain additional material such that the inflated shape will not achieve the basic shape directly, but will deflect outward between the radials and create a "sail". The MPCV canopy contains a small central vent, four rings with small gaps between each, and nine sails. There is a relatively large gap between sails one and two. Aside from the space created between sails two through nine by inflation and differing fullness, there are no explicit gaps. Eighty gores are sewn together circumferential to create the overall MPCV canopy.

Figure 1 shows two views of a single gore of the MPCV canopy. The rings and sails are alternatively colored white and orange for help differentiating them visually. Note the near zero fullness of the ring sections (4 panels at the top of 1) makes these panels appear as only the thickness of a line in the side view, while the fullness of sails is more evident as one moves away from the canopy centerline (top of 1 ). Here the difference in inflated shape between the bottom of a sail and the top of the adjacent sail results in a crescent shaped gap in the canopy. These gaps, along with the linear gaps between the ring section and between sails one and 2 , form the geometric porosity of the canopy.

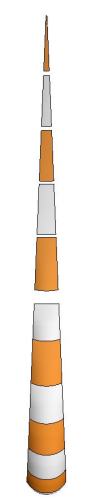

(a) Top

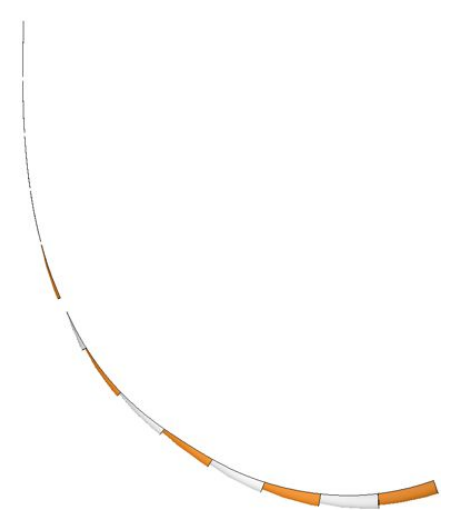

(b) Side View

Figure 1. MPCV canopy construction.

As the airflow through these gaps is important for the aerodynamic performance of the parachute, we believed finding a method that would generate geometries and correctly account for fullness and geometric porosity in the inflated MPCV main parachute, as well as new geometries for parametric analysis was very important. The Sandia National Labs' Canopy Loads Analysis (CALA) tool was chosen to generate these inflated shapes based on past experience using this tool. ${ }^{2}$ In addition, the CALA model of the Orion Main 
parachute already existed, reducing the time necessary to get to a CFD-ready geometry.

Inputs to the CALA code include the parachute geometric construction details, the material properties and stress-strain relations, weight of the suspended load, and the pressure-coefficient differential across the canopy material from the parachute center to the skirt. CALA iterates on dynamic pressure while computing the shape and stress/strain of a single canopy gore using structural analysis until the canopy drag equals the desired suspended load. One of the assumptions within CALA is that each of the canopy gores are identical, and as such it is limited to a symmetric canopy shape corresponding to steady descent at zero angle of attack. The input pressure distribution initially used for this analysis was based on parachute community experience, but during the analysis input from the CFD pressure distributions and evaluation of full-scale flight test geometry lead the authors to modify the distribution to more accurately reproduce the full-scale flight test geometry.

CALA outputs include the final inflated geometric information along with stress/strain information for each of the defined elements within the model. To generate a CFD grid from this output, a script was written to convert the CALA gore geometry output into a format directly useable in the overset grid generation process. This allowed the team to use any shape produced by CALA as a starting point for grid generation. These shapes could be used as produced by CALA directly, or the CALA output could be easily modified within the grid generation process in order to prescribe parametric variations. Figure 2 shows a comparison between an MPCV main parachute during a drop test and the output from the CALA code. Qualitative comparison of the CALA prediction of the fullness in the parachute sails and overall canopy shape is good.

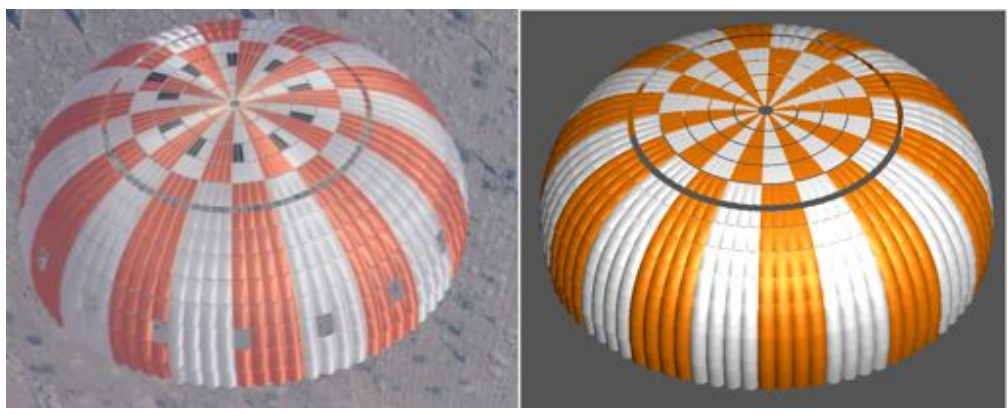

Figure 2. Comparison of parachute canopy from drop test (left) and CALA (right).

\section{Grid Generation}

With the large number of geometries considered for this work, the ability to quickly generate grid systems was important. Structured, overset grids of the parachute and the domain surrounding it were generated by using the Chimera Grid Tools (CGT) ${ }^{3,4}$ CGT allows for the creation of grid scripts for parametrically controlled grids for complex shapes. The script system holds fixed the surface and volume spacing, ensures bounded and consistent grid stretching ratios, and thus enforces grid-to-grid consistency - important when performing parametric studies as done here. Once these scripts are written, a new configuration can generally be gridded by simply changing the definition provided from CALA and re-running the scripts.

The PEGASUS5 domain connective software blanks portions of grids inside of solid bodies and provides interpolation stencils to provide communication between the overset grids. ${ }^{5}$ All of the grids generated for this work model only half of the complete parachute by means of a symmetry plane on the pitch plane to reduce computational cost. The grid systems showed variation in their final size, but generally had onehundred-million grid points with eighty-million in the parachute ring and sail grids. Figure 3 shows three cuts through the overset grid system for one canopy configuration.

Each ring or sail is gridded independently as a symmetric "o"-grid. Ideally, the canopy thickness would match the actual canopy materials, but to balance actual thickness with requirements for grid spacing and complexity, nine points were used to define the material edges using a finite thickness of 0.1 inches. Modeling these as thinner elements would require reducing the initial grid spacing on the sail surfaces, and would dramatically increases the number of grid points and expense for the computations. The reduced grid spacings could also create problems when generating the hyperbolic volume grids. With the use of this finite thickness, grid spacings on the resulting meshes vary smoothly on the surface of the rings and sails from 


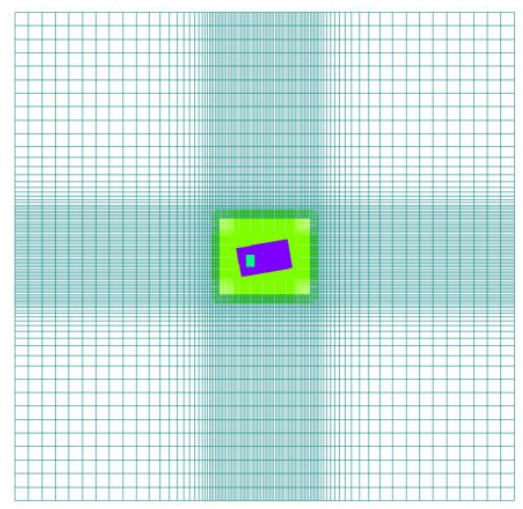

(a)

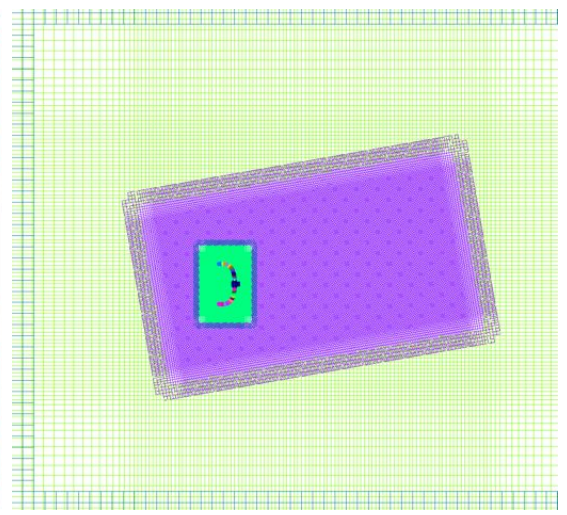

(b)

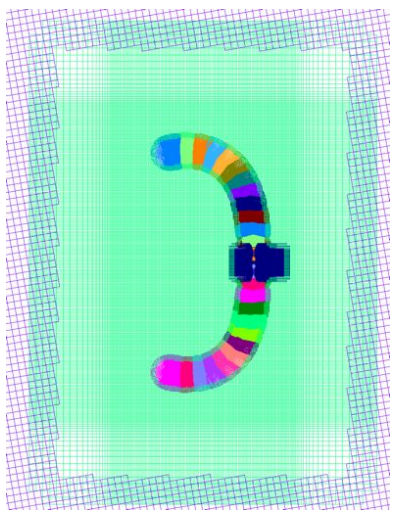

(c)

Figure 3. Illustration of computational grid topology.

leading to trailing edge, and there were no issues generating the volume grids. In parachute construction, the adjacent sails touch at the radial locations. For the computational grids, a 1.5" gap is included at the radial in order to allow for each sail and ring to be a separate grid with interpolation between adjacent ring/sail's volume grids.

In the volume, there are four major cartesian box grids. One is placed in the vent and allows for appropriate connectivity between the body-fitted grids at the center of the parachute (dark-blue in Fig. $3(\mathrm{c})$ ). A second, light-green in Fig. 3(c), surrounds the parachute with an isotropic spacing of $0.00432 D_{o}$ (normalized to the parachute reference diameter, 1390"). A third box, purple, is designed to capture the wake and is aligned to the freestream direction with an isotropic spacing of $.0129 D_{o}$ and extends $4.20 D_{o}$ behind the chute. Finally, an outer box surrounds the wake box and extends to the far-field boundaries (dark-green in Fig. 3(a)).

\section{Computational Methodology}

The computational fluid dynamic (CFD) simulations were performed with the OVERFLOW flow solver. It is a finite-difference, parallel, implicit Navier-Stokes' solver capable of using overset grid topologies. It is developed and maintained by NASA Langely Research Center. ${ }^{6}$

For this work, we have run the solver in a time-accurate mode with 10 Newton sub-iterations per time step. Central-differencing was used for discretization of the advective terms. Implicit time advancement used the diagonalized Beam-Warming scalar pentadiagonal scheme. Three levels of full-multigrid were used for flowfield initialization. Nearly all of the solutions were showed unsteadiness. Each solution was iterated until time-averaged integrated aerodynamics quantities became invariant with the inclusion of additional iterations. We found that a normalized time step of $0.00719\left(\frac{\Delta t V_{\infty}}{D_{o}}\right)$ was small enough to achieve temporally converged results for these non-moving cases.

This work employs the two-equation Shear Stress Transport turbulence model by Mentor. ${ }^{\text {With a RANS }}$ turbulence model, the wake of the parachute exhibits large, coherent vortices. These are not representative of the range of length scales that exist at flight Reynolds numbers, but they hopefully are representative of the bulk behavior to allow comparisons between cases to be valid.

The flight conditions for the MPCV main parachute are nominally Mach 0.03 at a dynamic pressure of about 1.3 [psf]. To avoid the use of low-Mach preconditioning and maintain numerical stability, the freestream velocity for the computations was modified to $165[\mathrm{ft} / \mathrm{s}]$. For a non-moving rigid canopy in incompressible flow, we do not expect this change to affect time-averaged aerodynamic results. Comparing several solutions of the flow solver at both conditions confirmed that our integrated forces and moments were unchanged by using these modified freestream conditions. 


\section{Results}

The work is divided into three parts: validating the tools against observed trends from Apollo drop test data, characterizing the stability of the current MPCV main parachute, and conducting parametric studies on geometric porosity in order to understand modifications' effects on static stability. Until more work is done to validate the magnitude of force coefficients seen in this analysis, we intend only to predict trends and relative magnitudes with this work. Import parameters for performance will be static stability and drag coefficient for the parachutes. Parachute forces were non-dimensionalized to a nominal parachute area of $10568 \mathrm{ft}^{2}$.

We infer static stability by the observing trends in the normal force coefficient, $C_{N}$, and assume that the behavior is a function of total angle of attack since the canopy is generally axisymmetric. The parachute canopy cannot rotate locally as long as the suspension lines remain in tension. Stability of the parachute behind the payload is then properly characterized by the pitching moment coefficient at the parachute attach point, $C_{m, A P} . C_{m, A P}$ is dominated by normal force due to the large riser plus suspension line length - which approaches nearly 2.0 times the parachute diameter.

\section{A. Validation}

Engineers developing the Mercury, Gemini, and Apollo decelerator systems conducted a large number of ground and flight tests in support program development. Initial validation of the CFD technique discussed above was performed against the data from the full-scale drop test program of the Apollo main parachutes. Detailed in a technical report are observations on the stability of several candidate Apollo parachutes as a function of changes in geometric porosity and other factors. ${ }^{7}$

The Apollo geometry was a ring-sail parachute with five rings and seven sails and in general had construction very similar to the MPCV main canopy. Three canopy shapes that mimic designs tested by the Apollo parachute improvement program are studied here in addition to the basic pre-modification ring-sail geometry as the baseline. Figure 4 shows canopy layouts for the baseline and modified parachutes. The modifications are as listed:

1. Removal of the geometric porosity in the crown (first 5 rings); the central vent remains.

2. Removal of 4 gores from the canopy, creating a conical geometry

3. Removal of 4 gores and removal of material to create a larger ring 5-to-sail 1 gap.

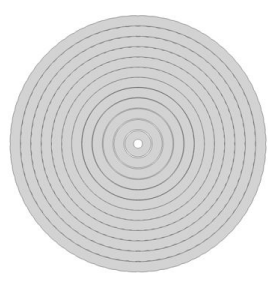

(a) Baseline

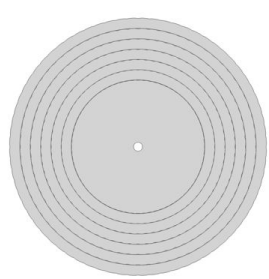

(b) Closed

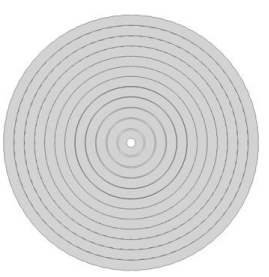

(c) Conical

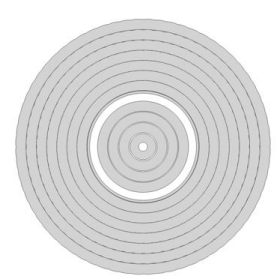

(d) Conical / Gap

Figure 4. Illustration of canopy layouts for Apollo main parachute modifications.

Figure 5 shows results from several Apollo drop tests. The plot is a histogram of the parachute system oscillation angle for the three designs. The baseline parachute and the conical mod are very similar in character. They show a near-uniform distribution of system oscillation angle, indicating that the parachute exhibited dynamic motion between 0 and nearly 30 degrees. In contrast, the inclusion of the $5 \%$ porosity gap shows improved performance, with system oscillation angles bounded to within 10 degrees of vertical.

Not shown in the figure is a histogram for the configuration which removed the ring geometric porosity. What is known from wind-tunnel testing is that the maximum oscillation seen by this canopy approached 


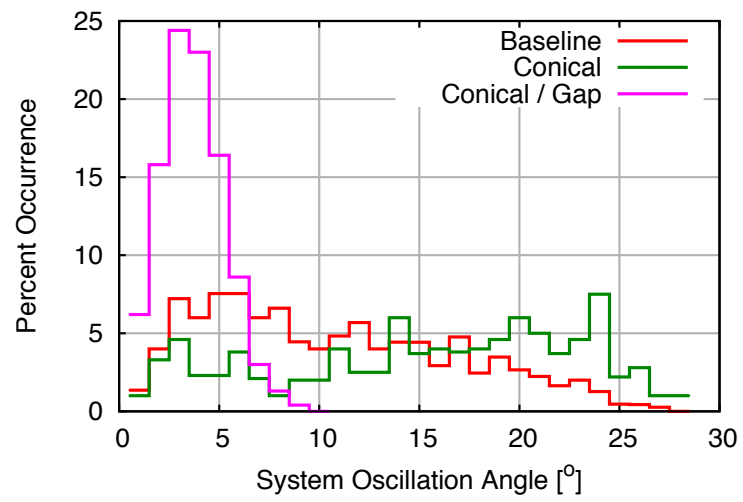

Figure 5. Histograms of system oscillation angle for several Apollo drop tests.

$30^{\circ} .8$ Of the three histograms, this is in family with the conical and baseline curves and the parachute stability is likely similar.

From these drop test results, it is clear that the large gap had the largest effect on increasing the stability of the parachute system. Closing the crown gaps and employing a conical modification may have changed the drop test behavior slightly, but they both show large swing angles in the data. To validate our approach with CALA and OVERFLOW, we show that the same trends are observed in the computational results of static stability.

To generate the updated canopy shapes, modified parachute CALA input models were created consistent with these changes. The new CALA models were then used to obtain predicted inflated shapes, and grids for OVERFLOW were made using the CGT scripts. Time-accurate CFD provided integrated force and moment predictions for each of the canopies.

Figure 6 show the integrated normal force from the CFD simulations. As the canopy is symmetric about the $\mathrm{Z}=0$ plane, only the solutions at negative $\alpha$ were computed and these results were mirrored across $\alpha=180^{\circ}$ to create the symmetric curve shown. In general, every $10^{\circ}$ in $\alpha$ were simulated in order to maximize throughput and infer gross trends.

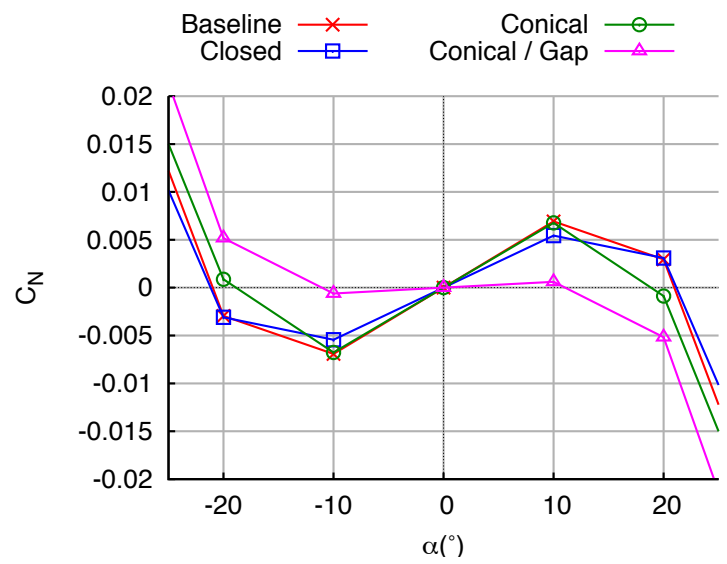

(a) Normal Force Coefficient

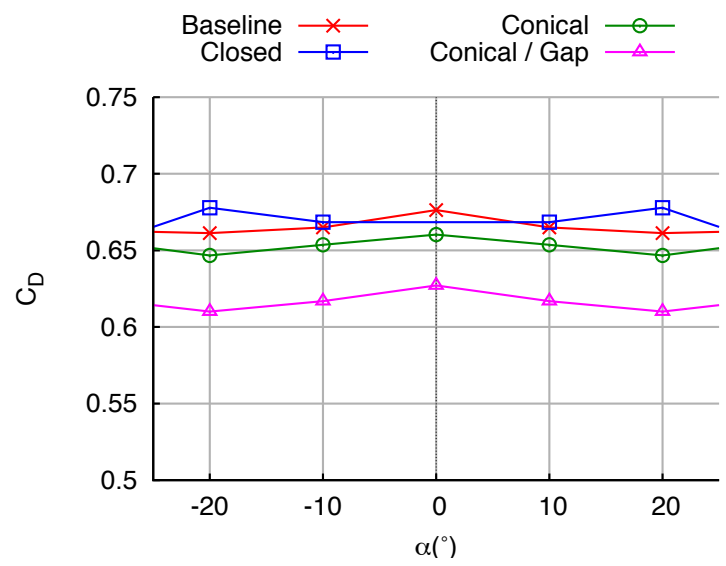

(b) Drag Coefficient

Figure 6. Integrated normal force and drag coefficients for modified Apollo main parachutes.

The normal force coefficient in Fig. 6 for the baseline Apollo geometry is unstable around $\alpha=180^{\circ}$. Similarly, the closed crown and conical configurations are predicted to be statically unstable. If static stability is a main contributor of overall system stability, this is consistent with the drop test results that showed these three canopies with large system oscillation angles. In comparisons, the conical configuration with the $5 \%$ porosity gap appears to be almost neutrally stable with a trim point close to $\alpha=10^{\circ}$.

For parachute canopies, increased porosity is typically associated with decreased drag. Our computational 
results confirm this trend. The canopy with the least porosity, the closed-crown canopy, has the highest drag while the one with the larger porosity, the $5 \%$ gap, has the lowest drag. The reference area for these coefficients is based on constructed diameter, so drag is directly proportional to the inflated projected area. For this reason, the baseline canopy has a slightly higher drag than the conical one. Apollo selected the 5\% gap to gain the stability and were able to tolerate the decreased drag. It's unlikely that the MPCV has the descent rate margin to add a similar about of porosity, but future detailed structural analysis will determine the envelope of allowable descent rates.

Additional validation of this technique against more quantitative data, such as wind tunnel test results is needed, but was not practical in the required timeframe. Assembling the data and constructing the CALA structural model for additional parachutes was determined to require too much time. This data is relevant to our work based on the similarity of the parachute design and the similar issues observed in drop tests. In addition, since the basic construction of the chutes is very similar - an approximation of the Apollo chutes could be obtained by easy changes to the MPCV CALA model.

These results are encouraging and are similar to what was observed experimentally. They illustrate that our approach might have success predicting trends in parachute stability. Moving forward, we use similar methods to explore possible modifications for encased stability for the MPCV main parachute.

\section{B. Stability of MPCV Main Parachute}

The final parachute used by the Apollo program did not see a reportable amount of pendulum in twoparachute cluster configurations. As was shown previously, the final Apollo main parachute was much more stable than some of the earlier designs. This helps support the theory that increased stability reduces the occurrence or magnitude of pendulum or eliminates it, and thus one possibility is that the MPVC main parachute, as currently designed is unstable and is contributing to the observed pendulum motion. To confirm this suspicion, simulations of the current main parachute were performed using the above-presented simulation technique.

Figure 7 shows canopy shapes for the current MPCV main parachute (a) and two modified canopies. The baseline parachute contains a nearly $2 \%$ geometric porosity slot between sails one and two. It also has missing panels in sail 7 every fifth gore and are referred here as 'windows'. The windows were added in an attempt to limit flyout and it is theorized that they also increase stability by increasing porosity. To quantify this effect, an baseline without the window cut-outs is also considered (Fig. 7(b)).

Also shown is a canopy without windows that includes a design option inspired by the Apollo program's final canopy. This is a $5 \%$ geometric porosity gap located at location of $0.4 h / h_{r}$ and expands the $2 \%$ gap present in the prior two configurations at the same location. Note that the MPCV main parachute has a diameter nearly one-third larger than the Apollo main parachute, nd it is unknown if a scaled slot will provide a similar trend in stability.

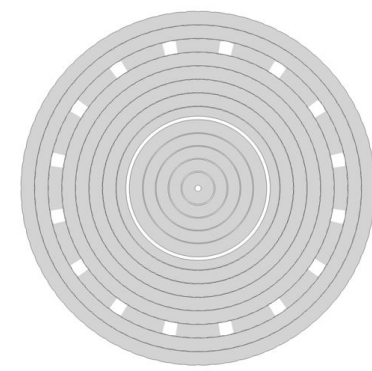

(a) Baseline

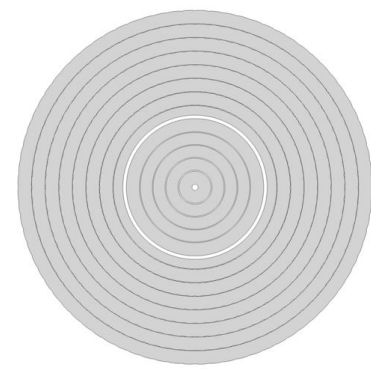

(b) No Windows

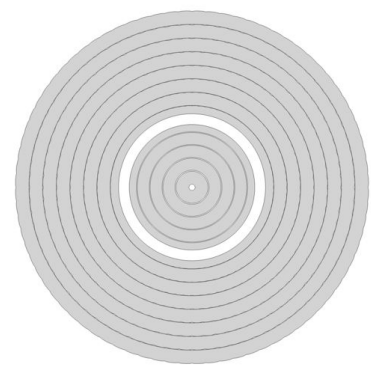

(c) No Windows / Apollo Gap

Figure 7. Illustration of canopy layouts for MPCV main parachute assessment.

Figure 8 shows the normal force and drag coefficients for the three canopies considered. Looking at the curve that corresponds to the baseline, it is clear that the normal force is unstable near $\alpha=0^{\circ}$. This instability may contribute to pendulum motion. Removing the windows creates an even less stable canopy with a larger (negative) normal force near $\alpha=-10^{\circ}$. The difference between the two identify a minor restoring contribution from the windows. 


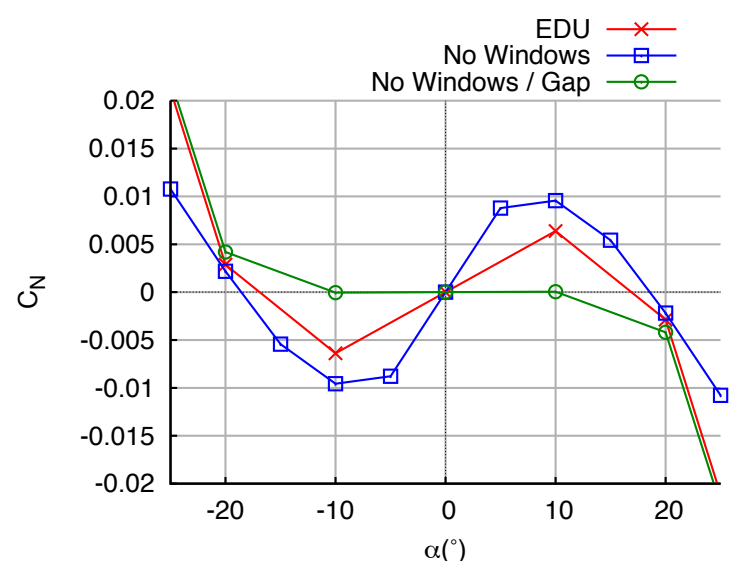

(a) Normal Force Coefficient

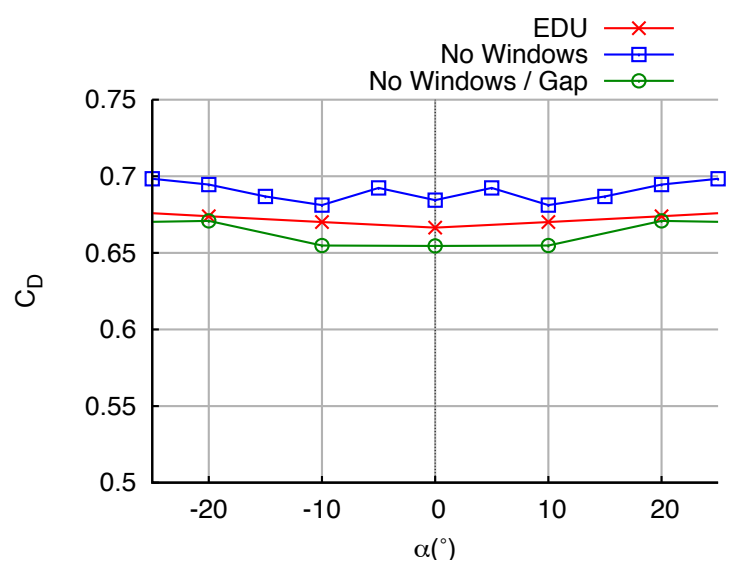

(b) Drag Coefficient

Figure 8. Integrated normal force and drag coefficients for modified MPCV main parachutes.

Similar to what the Apollo CFD simulations showed, adding a large $5 \%$ porosity gap in the MPCV parachute created an almost neutral stability near $\alpha=0^{\circ}$. This presents one obvious avenue for increased stability. However, there is an associated drag loss for each of the canopies that include additional porosity. Figure 8(b) quantifies the drag loss predicted by the CFD. In this case, the drag loss is less than $5 \%$ between the Baseline and the Apollo gap option.

The largest effect from the $5 \%$ gap is at $\alpha=10^{\circ}$. Figure 9 shows cumulative loads in the canopy from the vent to the skirt. Values attributed to each of the ring/sail elements are measurements of the cumulative load on the canopy from the element itself and all other elements between it and the vent. Each ring or sails's contribution to the normal force or drag is indicated by the jump between it's value and that of the next canopy element. Linear gaps built into the canopy such as the $5 \%$ gap noted above can be identified by the space between adjacent elements resulting in less-vertical jumps (see drag coefficient between sails one and two in Fig. 9(b)).

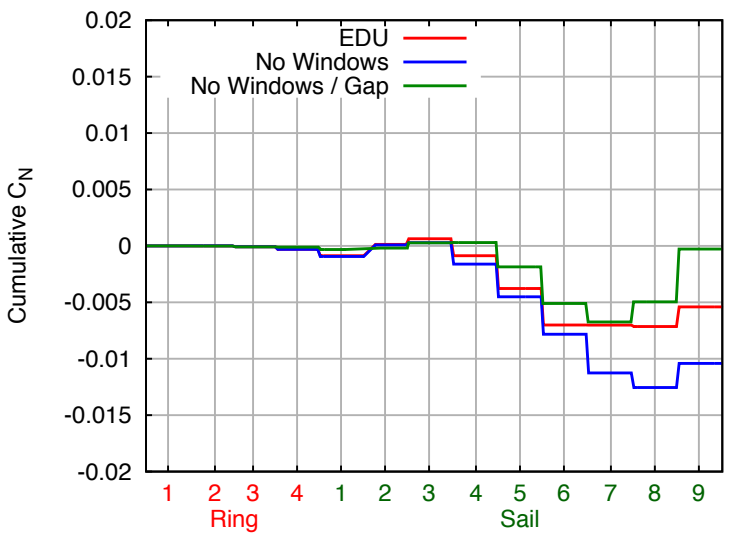

(a) Normal Force Coefficient

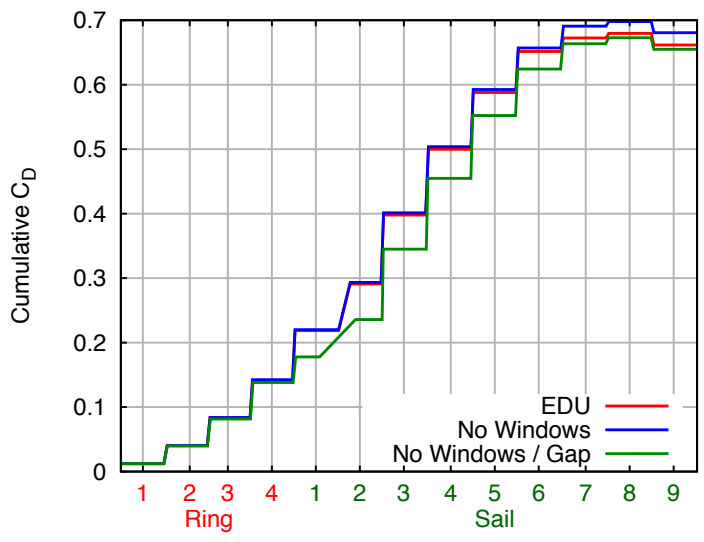

(b) Drag Coefficient

Figure 9. Cumulative normal force and drag coefficients for modified MPCV main parachutes; $\alpha=10^{\circ}$.

Effectiveness of the windows is apparent in the normal force coefficient. It serves to create a more symmetric pressure distribution and sails six through eight provide no additional normal force. This is in contrast to the canopy that does not include windows (blue) which sees a large negative, or unstable contribution from these sails. The effect from the large gap is very different. In normal force coefficient, there is little change between rings one and two at the location of the gap. Any loads created by this portion of the canopy are mostly in the axial direction since the surfaces are nearly perpendicular to the parachute's 
central axis. Near the skirt, the parachute with the $5 \%$ gap has a net stabilizing influence from sails seven, eight, and nine. The reason for this is discussed in greater detail in a subsequent section.

Figure 9(b) illustrates each portion of the canopy's contribution to overall drag. Even though the rings are relatively small in area, they are nearly normal to the freestream flow direction and as such contribute the majority of their forces in the drag direction. While considerably larger in terms of wetted area, the sails contribute less and less to drag as they become parallel to the flow near the skirt. The differences between the curves on the plot show that the $5 \%$ gap loses considerable drag between sails one and two due to the missing area. It does see a slight rise in the effectiveness of all subsequent sails, however. The windows only have a minimal effect on drag and only appear to influence performance on sail seven.

\section{Canopy Geometric Porosity Studies}

The team's proposed solution to the periodic swinging motion is increased static stability of the decelerator system. Constraining what can be done to achieve this stability increase is the need to maintain sufficient drag enabling the decelerator system to reduce descent velocity to acceptable levels. This necessitates solutions that increase stability but minimize the reduction in drag. In order to explore possible modifications, analysis studies on the MPCV main parachute were performed. The goal of these studies are to provide an understanding on what changes may be beneficial to arrest the pendulum action via increased static stability. Due to the current limitations of our tools, we are focusing only on modifications to geometric porosity and not material permeability.

Unlike the original Apollo and MPCV work, the parachute canopy shape was held constant while evaluating changes in geometric porosity. This means that CALA was run only once with the rings and sails from the original MPCV parachute modeled. The resulting inflated shape was modified with grid generation tools in order to create the porosity in the appropriate locations. In actuality, both the change in pressure distribution and the missing area would modify the force equilibrium within the canopy and influence the actual inflated shape. For these parametric analyses, we were interested in the effect of the porosity itself. While running CALA for each parametric variation would have been possible, the changes due to the increased porosity could be confused by canopy shape change. To completely evaluate canopy performance, the effects of both the porosity and the shape change would need to be considered. This overall canopy performance versus geometric porosity has not been studied with OVERFLOW at the current time, although CALA simulations of the configurations have been run to indicate relative drag performance.

\section{A. $5 \%$ Gap Locations}

The final Apollo configuration included at $5 \%$ porosity gap at a gore hight of $0.40 h / h_{r}$. It was not clear to the authors why this location was chosen, if there was something unique about its location, or if a similar amount of porosity at another location might achieve similar static stability. Figure 10 shows a collection of MPCV-size canopies that include a $5 \%$ porosity gap at different gore heights. The are of the gap remains constant, so for larger gore heights, the width of the gap becomes proportionally smaller.

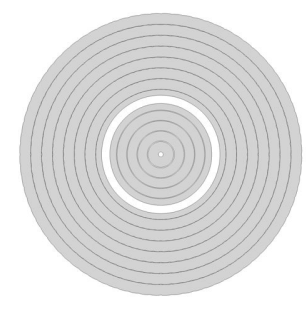

(a) $h / h_{r}=0.40$

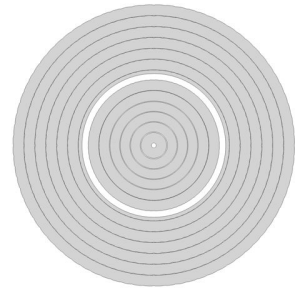

(b) $h / h_{r}=0.50$

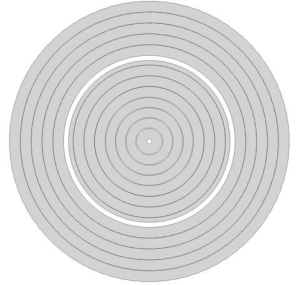

(c) $h / h_{r}=0.60$

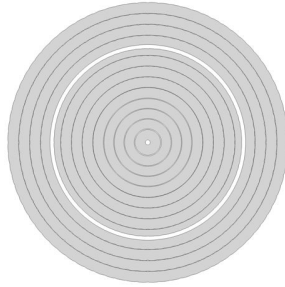

(d) $h / h_{r}=0.70$

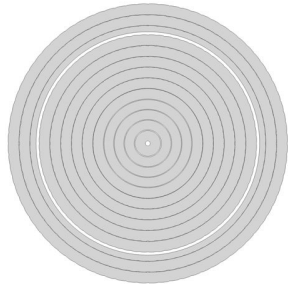

(e) $h / h_{r}=0.80$

Figure 10. Illustration of canopy layouts for $\mathbf{5 \%}$ gap location cases.

Figure 11 shows the integrated forces on these canopies and the results from a canopy without any gap as a reference. In terms of static stability, the gaps at 0.40 and $0.50 h / h_{r}$ show similar performance and neutral stability. Gaps at $0.60,0.70$, and $0.80 h / h_{r}$ are unstable around $\alpha=0^{\circ}$, but has a much stronger 
restoring force at higher $\alpha$ with higher $h / h_{r}$ providing a greater force. All canopies with a $5 \%$ gap are less unstable than the no-gap comparison.

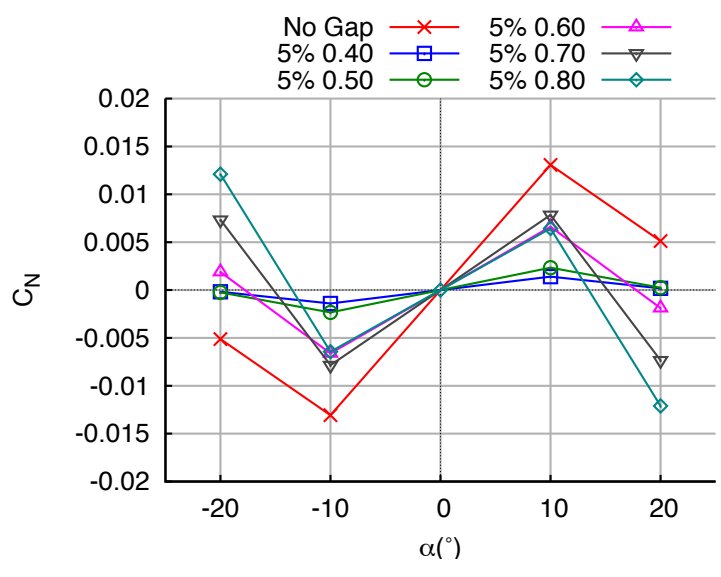

(a) Normal Force Coefficient

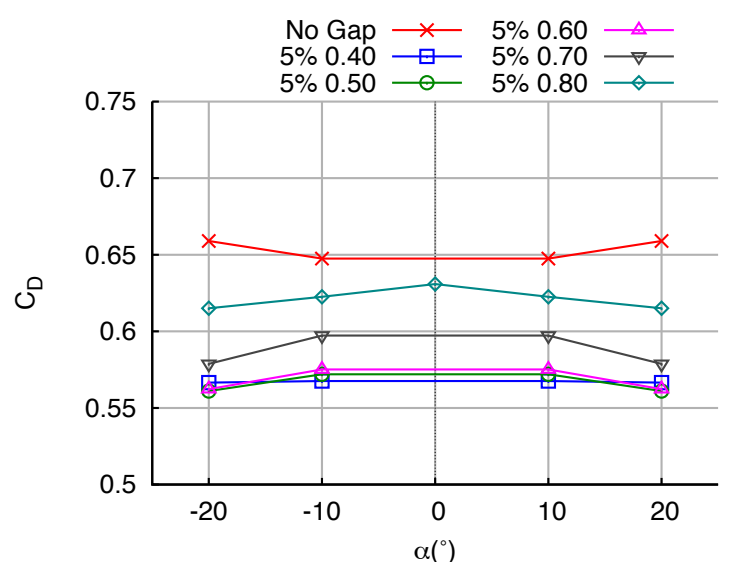

(b) Drag Coefficient

Figure 11. Integrated drag and normal force coefficients for varying location $5 \%$ gap.

Figures 12 and 13 show cumulative loads for these parachute canopies.

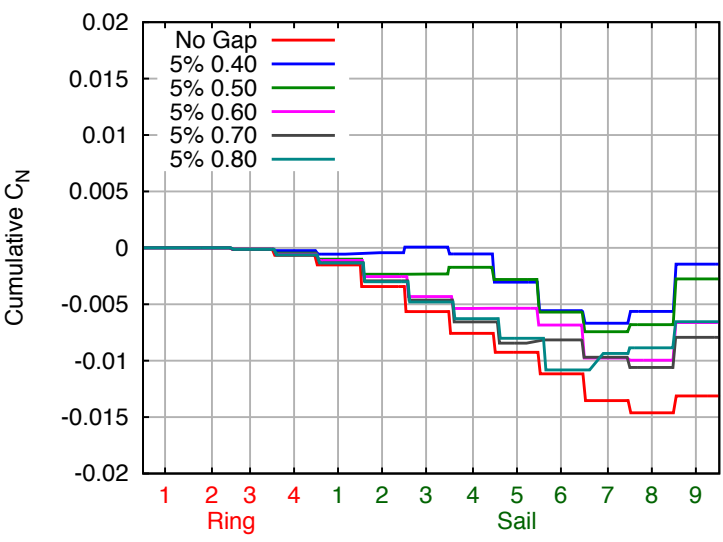

(a) Normal Force Coefficient

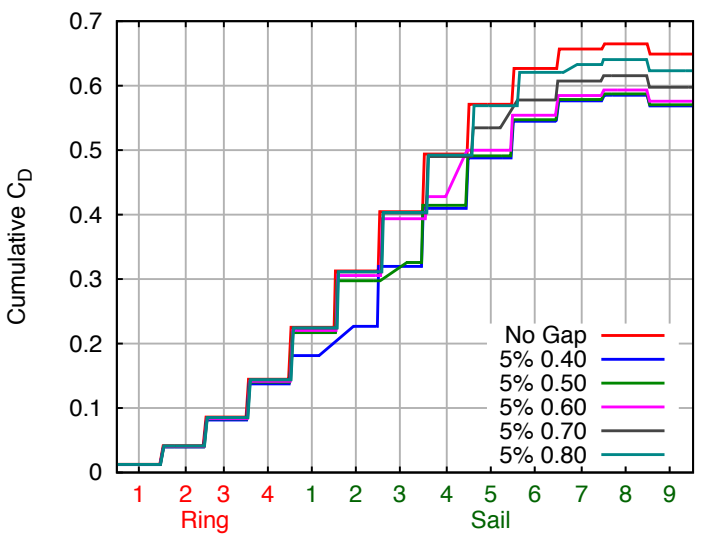

(b) Drag Coefficient

Figure 12. Cumulative normal force and drag coefficients for varying location $5 \%$ gap; $\alpha=10^{\circ}$.

The results for drag coefficient are mixed. All of the canopies with the gap have a large drag deficit compared to the no-gap canopy. It appears that there is a slight preference for gap locations near 0.70 or 0.80 for increased drag with a $5 \%$ porosity gap. One reason for this trend is that the canopies are curved. The force acting on the area of the parachute that is removed by the gap is not as closely aligned with the drag vector. This result highlights the fact that our method is holding the canopy shape fixed, and does artificially increase the diameter of the canopies with gaps at high gore heights.

\section{B. Porosity at $0.40 h / h_{r}$}

Since both the MPCV and Apollo programs have a geometric porosity created by a slot at a gore height of $0.40 h / h_{r}$, this location was selected to parametrically vary the slot size and thus geometric porosity. Increased porosity, from $2 \%$ to $5 \%$, changed the character of the predicted stability for the MPCV main parachute to be neutrally stable. Figure 14 shows parachute canopies with a varying amount of porosity at $040 h / h_{r}$. Porosity was added in $1 \%$ increments from $1 \%$ to $5 \%$. Simulations with these designs should help provide understanding for the the change in character exists. 


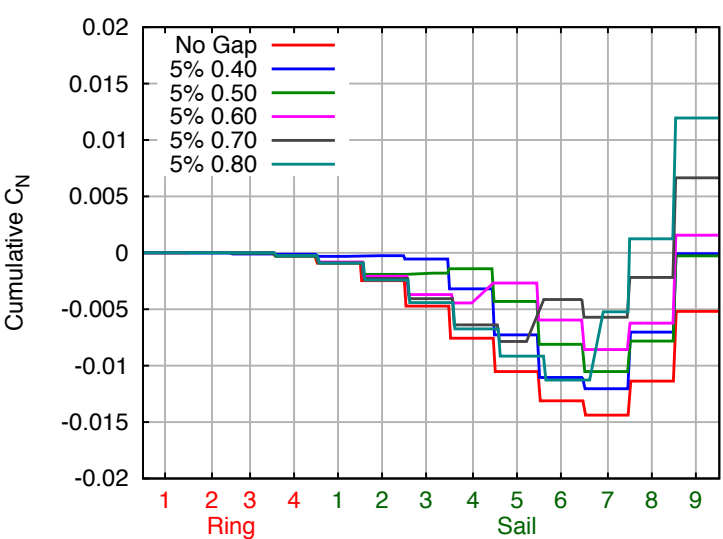

(a) Normal Force Coefficient

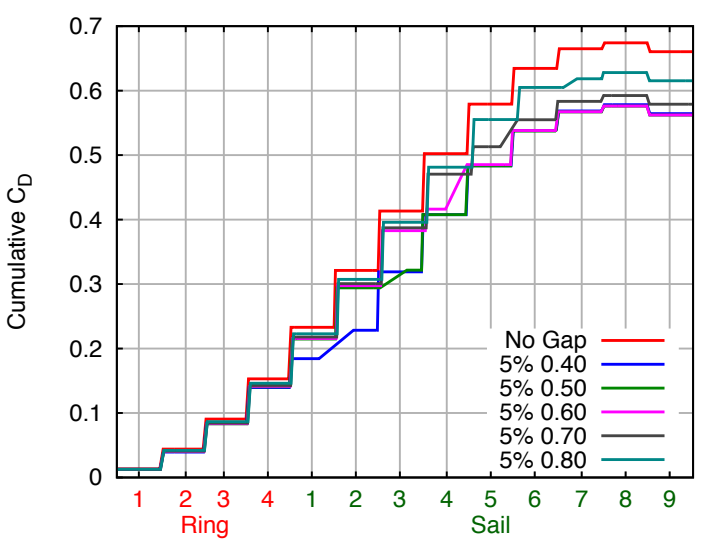

(b) Drag Coefficient

Figure 13. Cumulative normal force and drag coefficients for varying location $5 \%$ gap; $\alpha=20^{\circ}$.

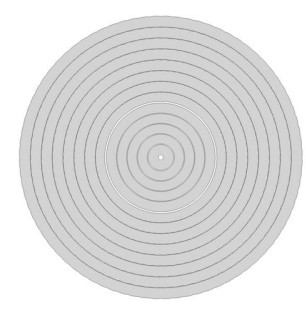

(a) $1 \%$ Porosity

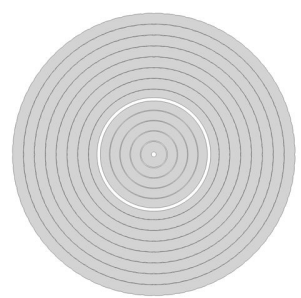

(b) $2 \%$ Porosity

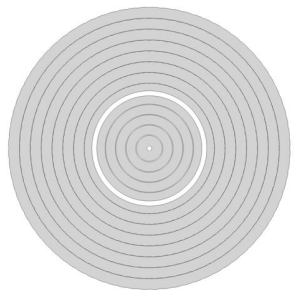

(c) $3 \%$ Porosity

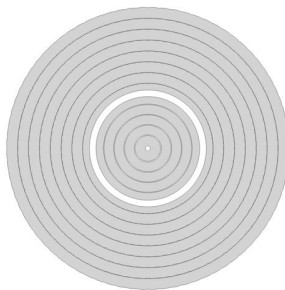

(d) $4 \%$ Porosity

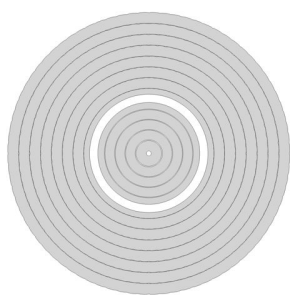

(e) $5 \%$ Porosity

Figure 14. Illustration of canopy layouts for case with varying porosity at $0.40 h / h_{r}$.

The integrated normal force coefficients for these simulations show an interesting result. For the parachutes with less than $3 \%$ geometric porosity, there is a trend for instability near $\alpha=0^{\circ}$. However, for all parachutes with a geometric porosity of $4 \%$ or greater, our method predicts neutral stability as shown in Figure 15 . The changes to the stability for this configuration are largest at $\alpha=10^{\circ}$ with smaller effects at $20^{\circ}$, but this may be due only to the fact that the no-gap configuration showed a large unstable moment at $\alpha=10^{\circ}$ and only a small unstable moment at $20^{\circ}$.

Trends in the drag coefficient do not show this non-linear trend. Instead, there is a very normal progression of drag that is almost directly related to the increase in porosity. This threshold effect is interesting because it implies that modifying the MPCV parachute by only slightly increasing the size of the current gap could dramatically improve performance, but at the same time we are not confident in our tools to predict a value of porosity required for this change.

To better understand the cause for the sharp change in behavior between $2 \%$ and $3 \%$ porosity, images of the time-averaged flowfield are insightful. Figure 16 show images of velocity contour in the pitch plane for the canopies. In addition to velocity magnitudes, the images also show bulk flow structure by means of line integral convolution (shown as highlighted streamlines). In all of the figures, the large, separated wake is pronounced. As is the 'bubble' of captured air ahead of the canopy.

As the geometric porosity at $0.40 h / h_{r}$ is increased, the flow emanating form the inside of the canopy grow larger and more powerful. At 3\% porosity and higher, the leeward side of the high-speed flow is strong enough that it is able to push the dominant RANS wake away from the canopy and shields the leeward surface. Without this recirculating flow impinging on the canopy, there is locally lower pressure and an increased normal force. Increasing the porosity further provides a more pronounced plume, but it is the interference with the main wake the provides the improved static stability.

Loading through the canopy is shown in Fig. 17. 3\% and higher see the same effect that we saw earlier with the MPCV EDU comparisons. Sails seven through nine are very effective at creating a stabilizing 


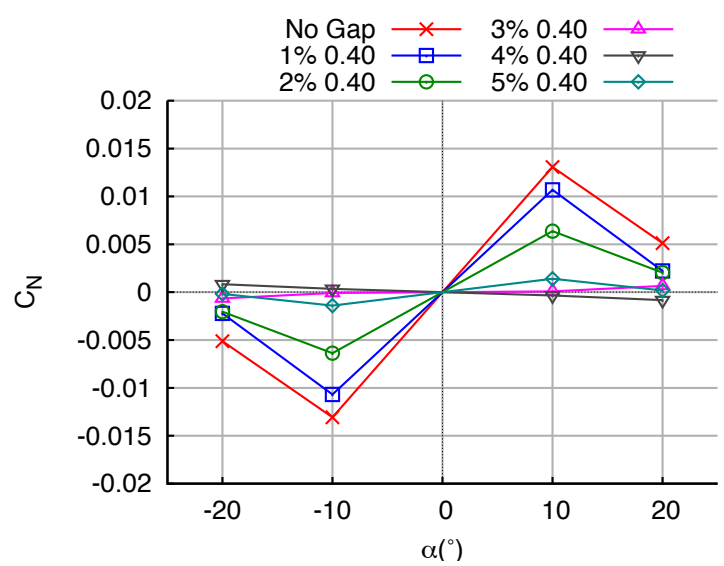

(a) Normal Force Coefficient

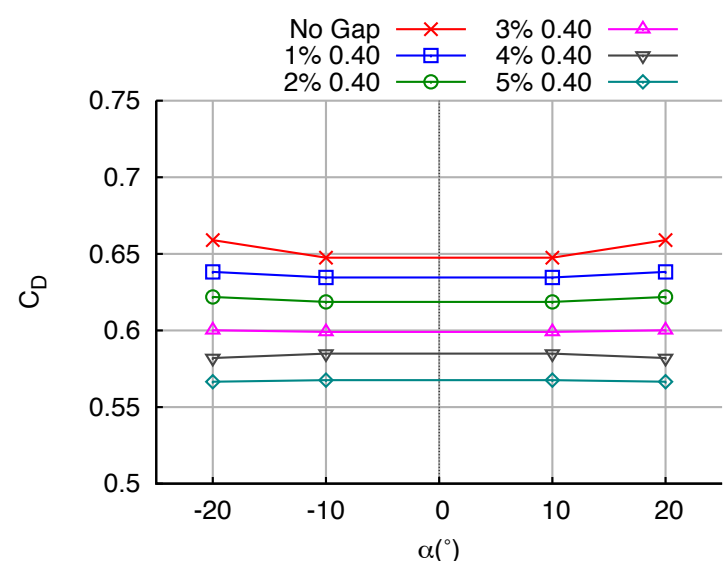

(b) Drag Coefficient

Figure 15. Integrated drag and normal force coefficients for varying gap sizes at $0.40 h / h_{r}$.

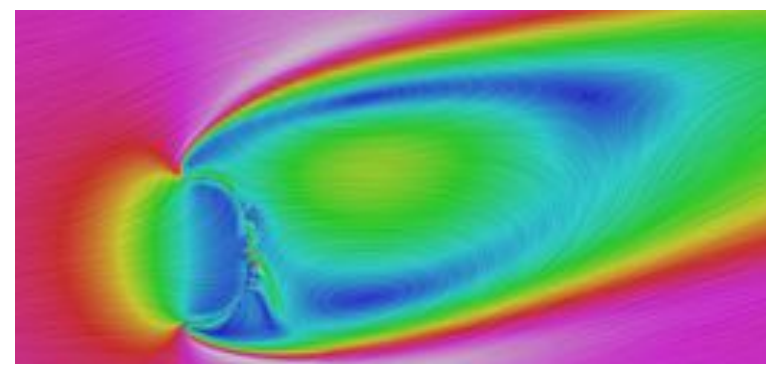

$0 \%$ Porosity

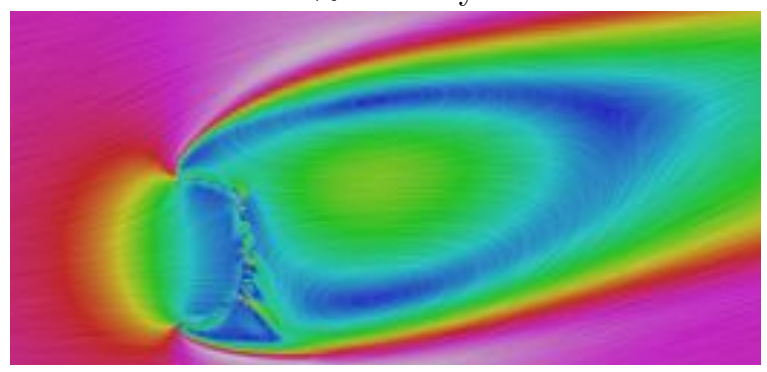

$2 \%$ Porosity

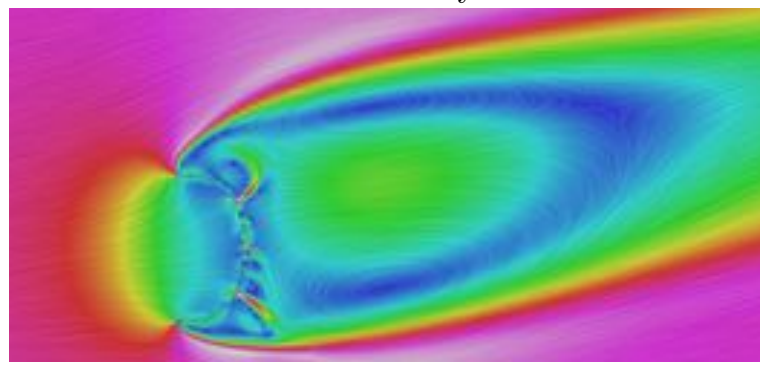

$4 \%$ Porosity

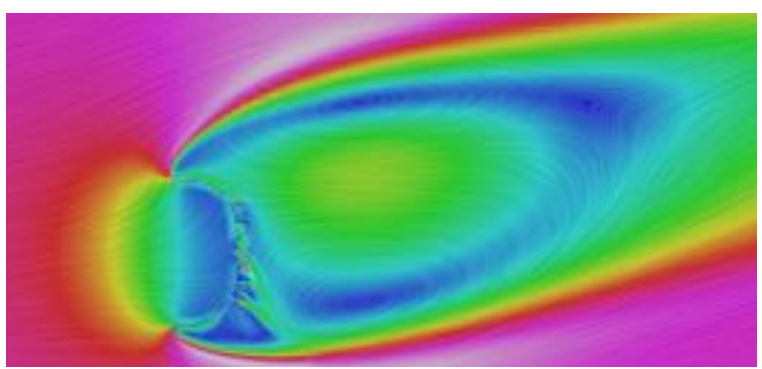

$1 \%$ Porosity

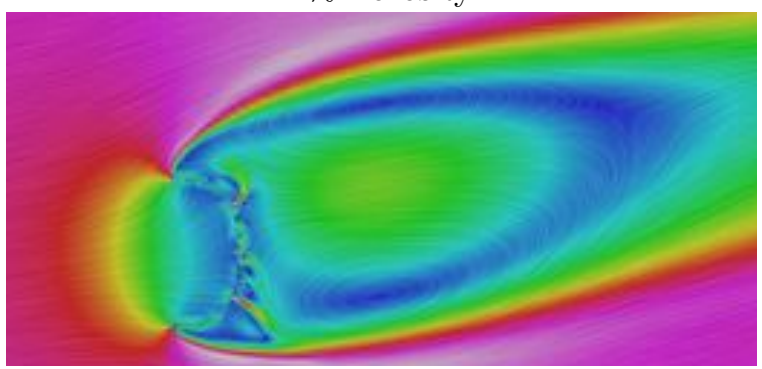

$3 \%$ Porosity

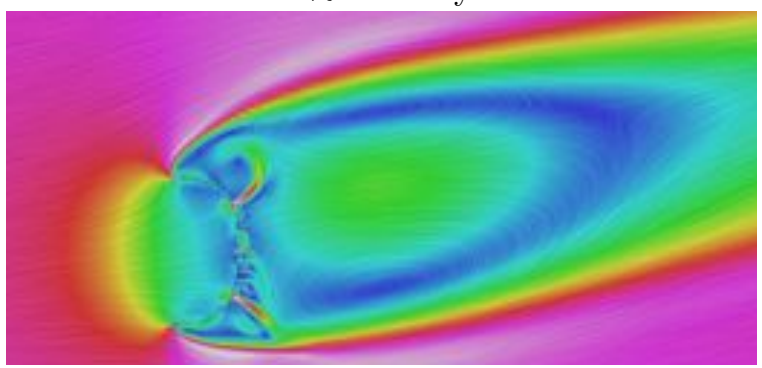

$5 \%$ Porosity

Figure 16. Effect of geometric porosity at $h / h_{r}=0.40$ on predicted wake at $\alpha=10^{\circ}$.

normal force when shielded from the dominant wake. Smaller effects are seen in the same region for $1 \%$ and $2 \%$ porosity. Drag is almost linearly related to porosity by the loss between sails one and two at the gap location. 


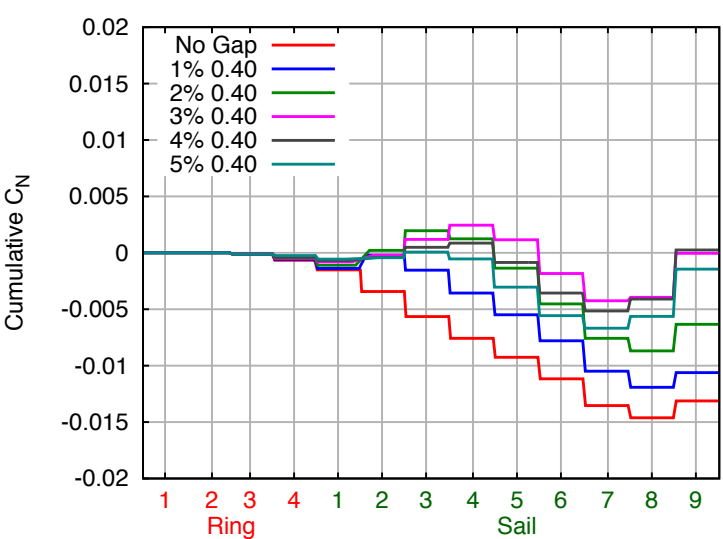

(a) Normal Force Coefficient

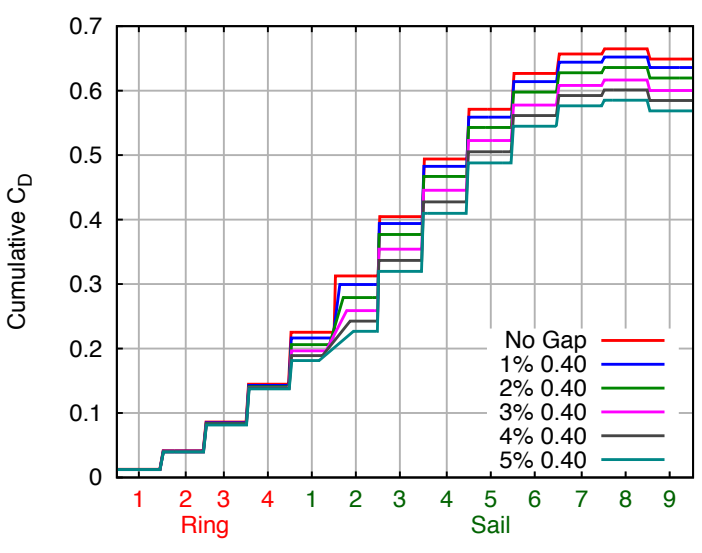

(b) Drag Coefficient

Figure 17. Cumulative normal force and drag coefficients for varying gap sizes at $0.40 h / h_{r} ; \alpha=10^{\circ}$.

\section{Porosity at $0.80 h / h_{r}$}

Previously, geometric porosity near the skirt showed an opportunity to greatly increase the restoring force at high- $\alpha$ without incurring as large of a loss in drag. The current design for the MPCV main parachute has window cut-outs that provide a total geometric porosity of $2.45 \%$ and are centered at $0.81 h / h_{r}$. Results to date indicate that replacing them with a constant porosity ring may create a more stable canopy.

A second parametric study of geometric porosity near $0.80 h / h_{r}$ was preformed to determine trends and understand if there was a minimum effective gap similar to what was seen with the suite at $0.40 h / h_{r}$. Figure 18 shows the layouts considered for these cases. A $2 \%$ porosity gap showed little effect, so a $1 \%$ gap was not investigated. Compared to the windows in the MPCV parachute, a 5\% porosity gap is quite large.

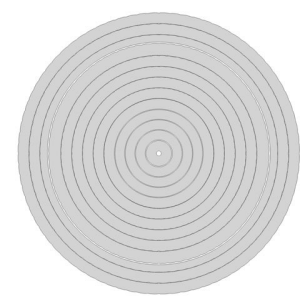

(a) $2 \%$ Porosity

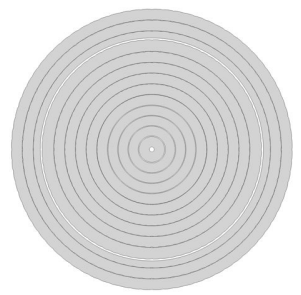

(b) $3 \%$ Porosity

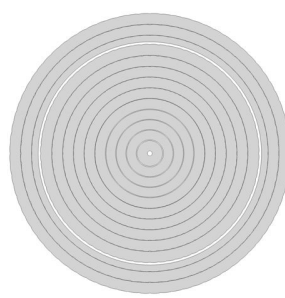

(c) $4 \%$ Porosity

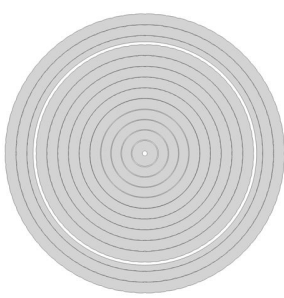

(d) $5 \%$ Porosity

Figure 18. Illustration of canopy layouts for case with varying porosity at $0.80 h / h_{r}$.

Figure 19 shows the integrated aerodynamic results for this study. The normal force coefficient shows improved stability additional porosity, and shows a general increase in effect as the porosity is increased. One might argue that there appears to be a change in character between $3 \%$ and $4 \%$ for $\alpha=20^{\circ}$ but at $10^{\circ}$ no change in character is readily apparent and at for both attitudes the stability benefit continues to increase up to the $5 \%$ value studied. Unlike the results at $0.4 h / h_{r}$, increases in porosity here influence the $20^{\circ}$ stability in a significant manner. Drag coefficient results show a slight drop with the addition of a gap, but remains largely constant as porosity is increased to $5 \%$.

Figure ?? shows a set of velocity contour plots in the pitch plane for varying porosity at $0.80 h / h_{r}$. Similar to the behavior seen with the gap at $0.40 h / h_{r}$, the gap modifies the local flow and tends to isolate it from the influence of the predominate RANS wake, but unlike the images shown in Fig. 16, the flow behavior is more progressive as porosity is increased, and does not show an overall step change in character.

Canopy line-loads for both Normal and Drag coefficients at $10^{\circ} \alpha$ are shown Fig. 21. In Normal force, with the exception of the canopy without a gap, the performance of the rings sails up to Sail 6 are extremely similar. For Sails 7-9, there is a progressive change in the normal force coefficient behavior toward more 


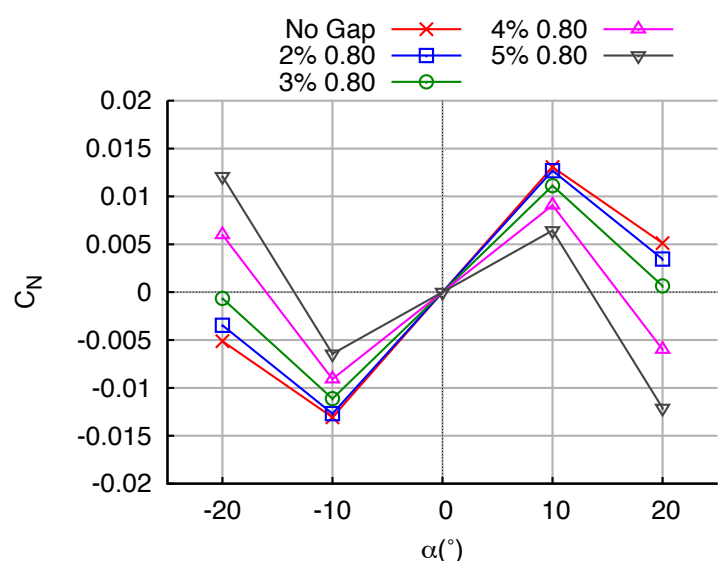

(a) Normal Force Coefficient

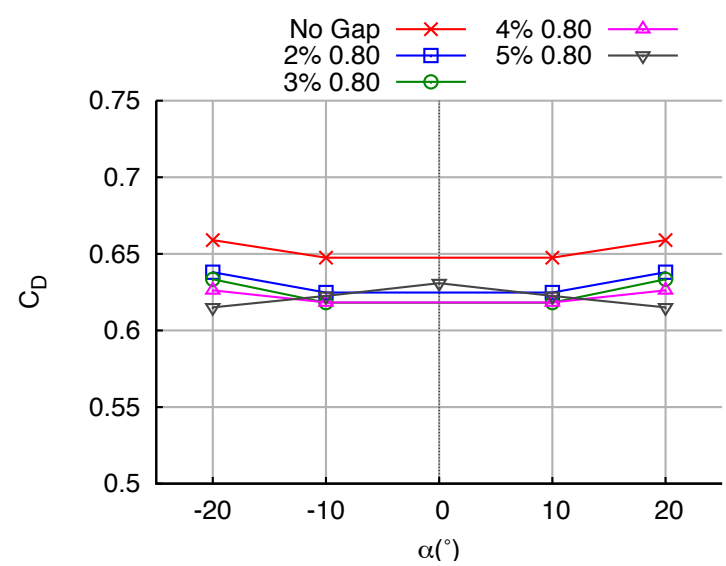

(b) Drag Coefficient

Figure 19. Integrated drag and normal force coefficients for varying gap sizes at $0.80 h / h_{r}$.

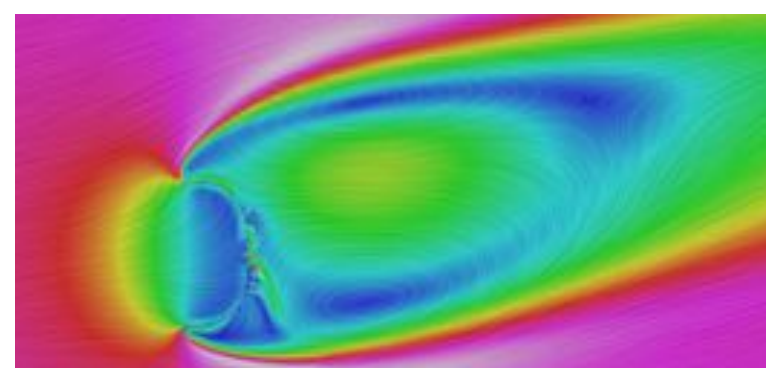

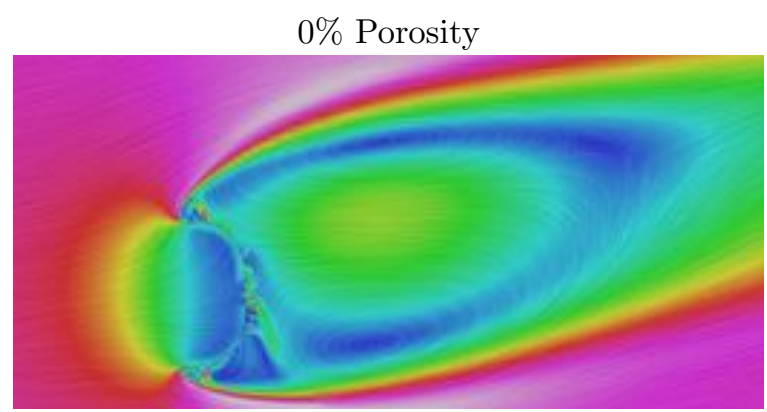

$2 \%$ Porosity

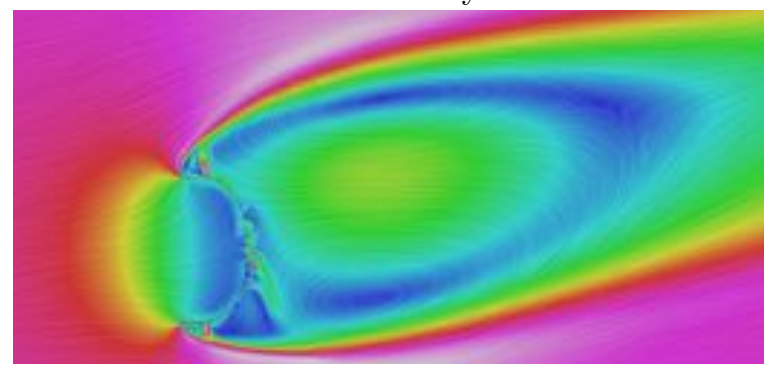

$4 \%$ Porosity

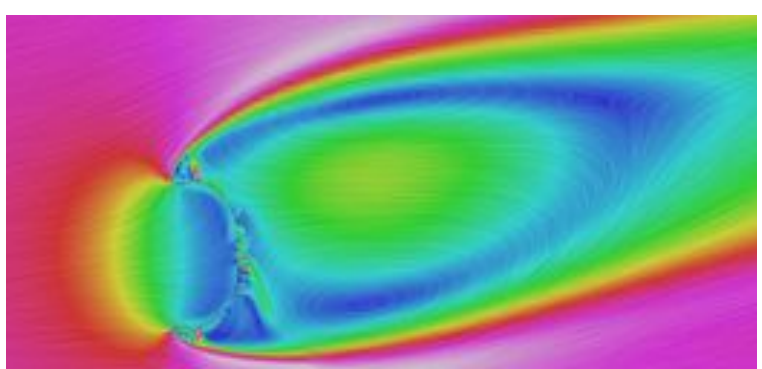

$3 \%$ Porosity

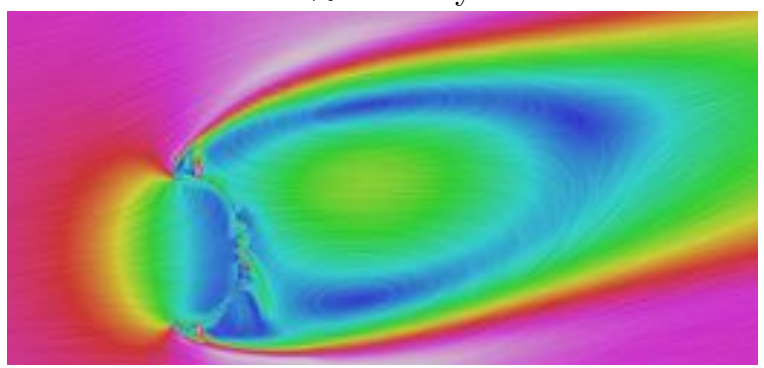

$5 \%$ Porosity

Figure 20. Effect of geometric porosity at $h / h_{r}=0.80$ on predicted wake at $\alpha=10^{\circ}$.

stability. Interestingly - the $2 \%$ gap achieves only slightly improved stability to the no gap model, but where sail 9 contributes a positive stability increment with no gap, this positive increment is not present in the $2 \%$ gap (or the 3\%) configuration. At 3\%, the static stability contribution of Sails 7-9 is neutral, and becomes increasingly positive as the porosity is increased to $5 \%$. 
Drag performance of all of these configurations is very similar up until sail 7, where the drag of all configurations with a gap departs from the no-gap results. All of the configurations with a gap are very similar regardless of the porosity value, as expected given the integrated aerodynamic results.

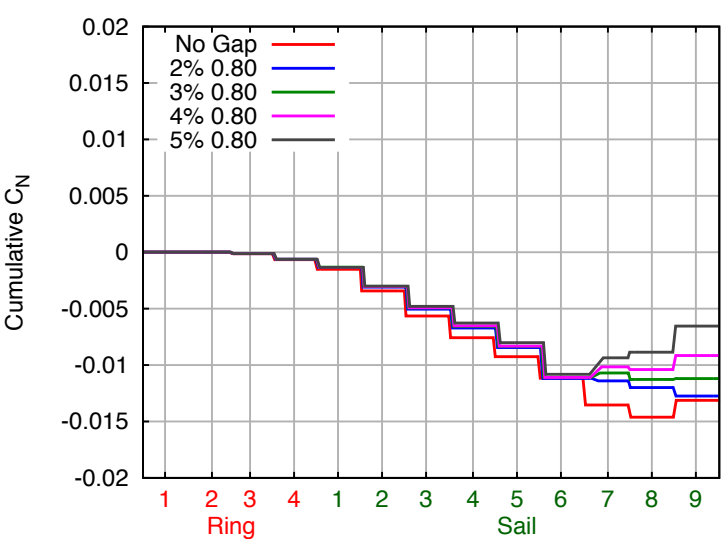

(a) Normal Force Coefficient

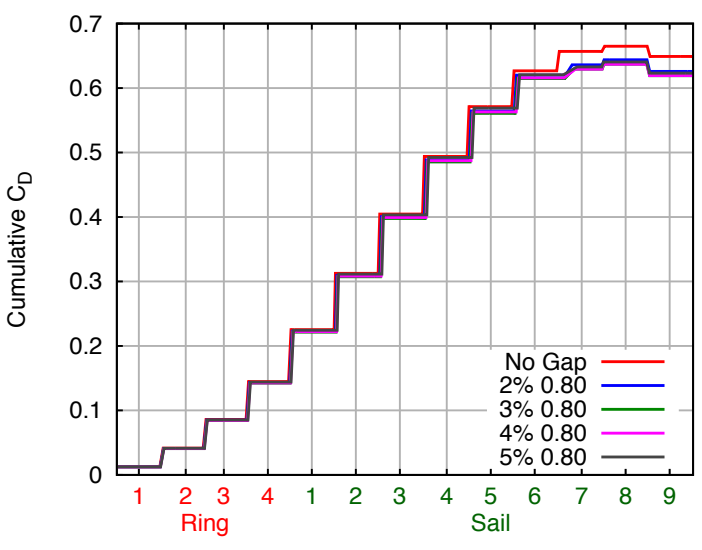

(b) Drag Coefficient

Figure 21. Cumulative normal force and drag coefficients for varying gap sizes at $0.80 h / h_{r} ; \alpha=10^{\circ}$.

As flow incidence angle is increased to $20^{\circ} \alpha$ the line-load trends differ from those at $10^{\circ} \alpha$ in that differences in the Drag coefficient are evident between the configurations starting as early as Ring 4 . If the $1 \%$ gap case had been run, one could envision it fitting between the drag results of the no-gap and the $2 \%$ gap. Normal force coefficient here is less well behaved, but in a gross sense the majority of the changes between canopy configurations occurs on sail 7-9 with effects on the lower numbered sails more evident as porosity is increased. The $2 \%$ porosity curve is the outlier here, with sail 7 not behaving in-family with the other increased porosity configurations, but excepting this one sail and porosity value, all of the remaining sail 7-9 panels similar trends with an increasing magnitude of stability as porosity is increased.

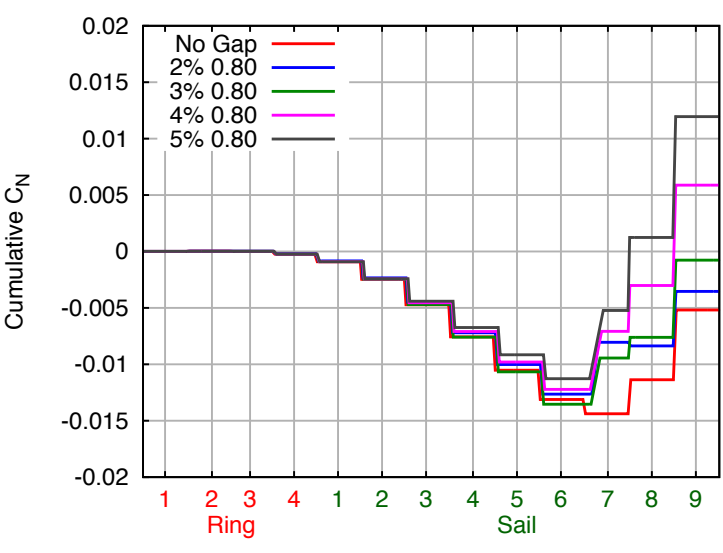

(a) Normal Force Coefficient

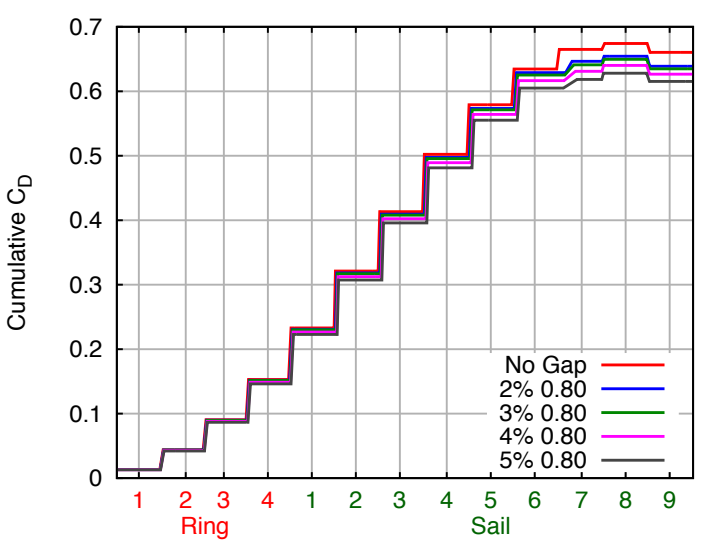

(b) Drag Coefficient

Figure 22. Cumulative normal force and drag coefficients for varying gap sizes at $0.80 h / h_{r} ; \alpha=20^{\circ}$.

\section{Porosity at 0.40 and $0.80 h / h_{r}$}

The parametric study results discussed previously showed that a geometric porosity between $2 \%$ and $3 \%$ located at $0.40 h / h_{r}$ created a relatively large improvement in normal force for $\alpha=10^{\circ}$. The porosity at $0.80 h / h_{r}$ tended to effect both $\alpha=10^{\circ}$ and $\alpha=20^{\circ}$ but with the larger effects occurring at $20^{\circ}$. Canopies with gaps at both locations could combine the advantages of the two. 
At this point in our analysis, feedback from the parachute team included concerns about the effects on inflation and reefed stage loads (where dynamic pressure is significantly higher than during full-open operations) that changes at $h / h_{r}$ of 0.5 or less might have. As result, for the next analysis set, the baseline $2 \%$ gap from the MPCV main parachute is used at $0.40 h / h_{r}$ and a range of gap sizes are studied at 0.80 $h / h_{r}$. While $2 \%$ porosity at $0.40 h / h_{r}$ did not reach the plateau of stability, it does show advantages over zero porosity, and it was hoped that these effects would combine with the benefits of adding a continuous gap at $0.80 h / h_{r}$. For comparison, the EDU configuration with window cut-outs in sail 7 is included - recall that the windows add a total porosity of approximately $2.5 \%$. Figure 23 shows the canopies that are examined in addition to a baseline without any porosity at $0.80 h / h_{r}$.

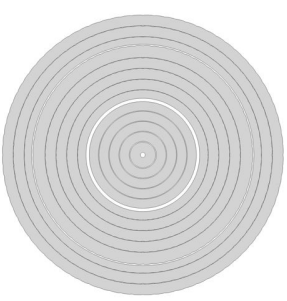

(a) $2 \%$ Porosity

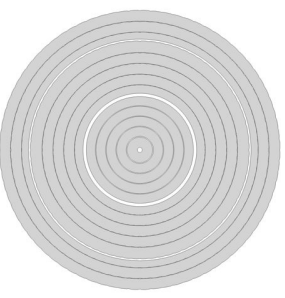

(b) $3 \%$ Porosity

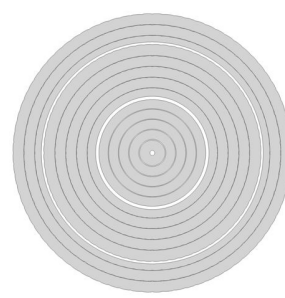

(c) $4 \%$ Porosity

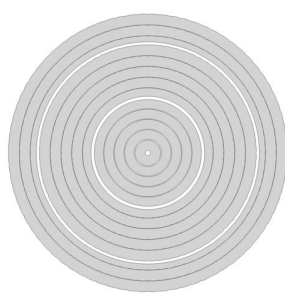

(d) $5 \%$ Porosity

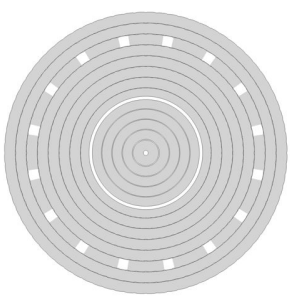

(e) Windows

Figure 23. Canopy diagrams with varying porosity at $0.80 h / h_{r}$ and $2 \%$ porosity at $0.40 h / h_{r}$.

The integrated normal force coefficient values shown in Fig. 24 show the improvement approaches simple superposition of the previous independent results. Compared to the cases with gaps only at $0.40 h / h_{r}$, there is much improved stability at higher angles of attack. At $\alpha=10^{\circ}$, there is also improved static stability when compared to canopies with porosity added only at $0.80 h / h_{r}$. The drag loss between the canopies is similar to what was seen in the sweep at $0.80 \mathrm{~h} / \mathrm{h}_{r}$.

It is interesting to compare the configuration using discrete windows to the continuous slot porosity. Recall the windows are approximately $2.5 \%$ geometric porosity, so the windows produced equivalent stability, the normal force should fall between the $2 \%$ and $3 \%$ results. The integrated aerodynamics however show the windows are outperformed even by the $2 \%$ results at $\alpha=20^{\circ}$ and has similar results for $\alpha=10^{\circ}$. In terms of normal force coefficient, the concentric ring of porosity appears to provide a much more effective means of providing static stability for all angles of attack. Drag values do appear to be slightly superior for the window configuration however, but are within a percent or two.

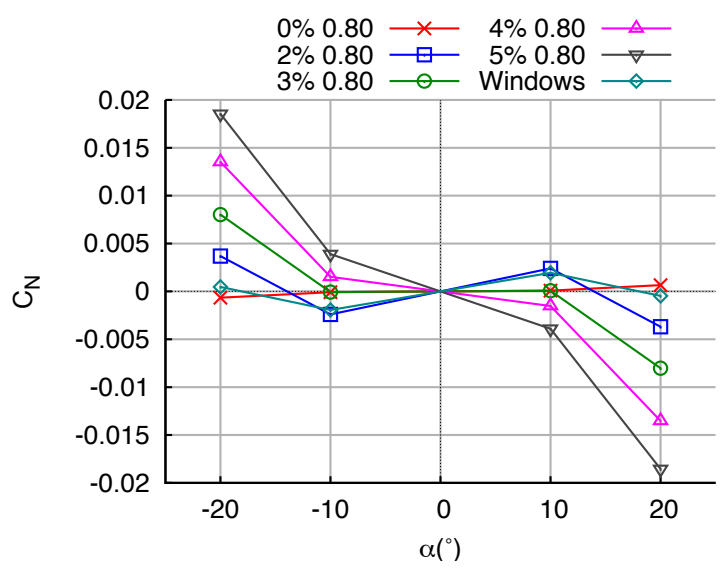

(a) Normal Force Coefficient

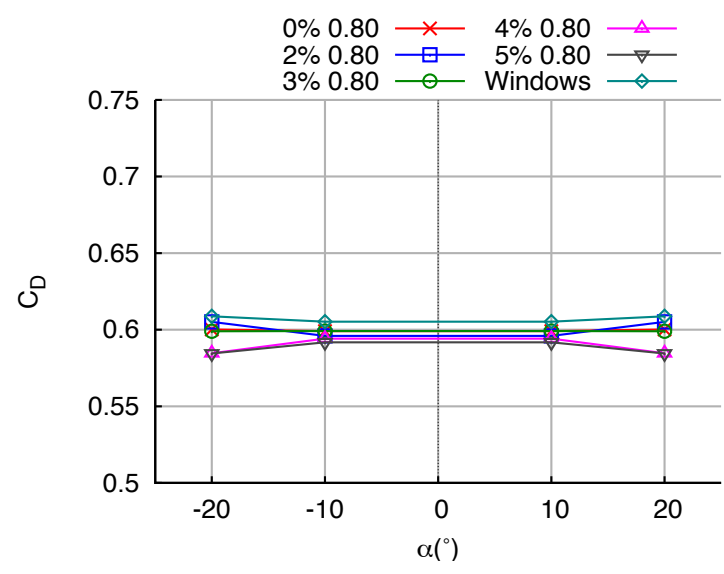

(b) Drag Coefficient

Figure 24. Integrated drag and normal force coefficients for a varying gap sizes at $0.80 h / h_{r}$ with gap at 0.40 $h / h_{r}$.

Figures 25 and 26 show line loads for these canopies at $10^{\circ}$ and $20^{\circ} \alpha$.

Initial conversations with the Orion MPCV parachute team have indicated strong preference to not 


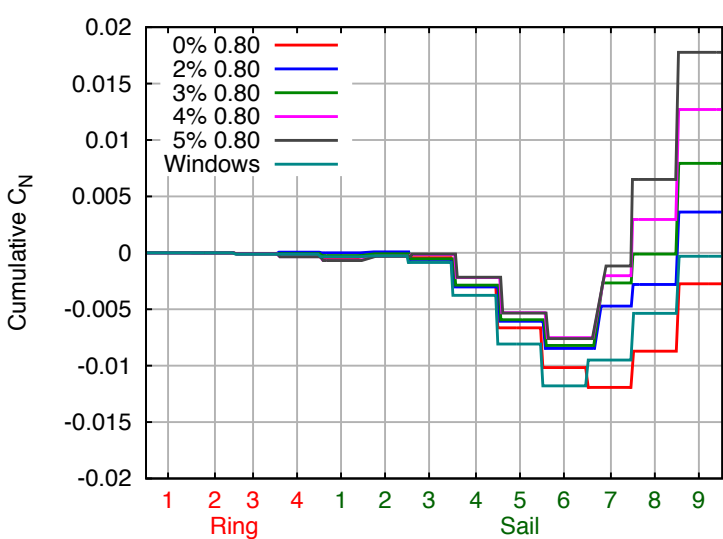

(a) Normal Force Coefficient

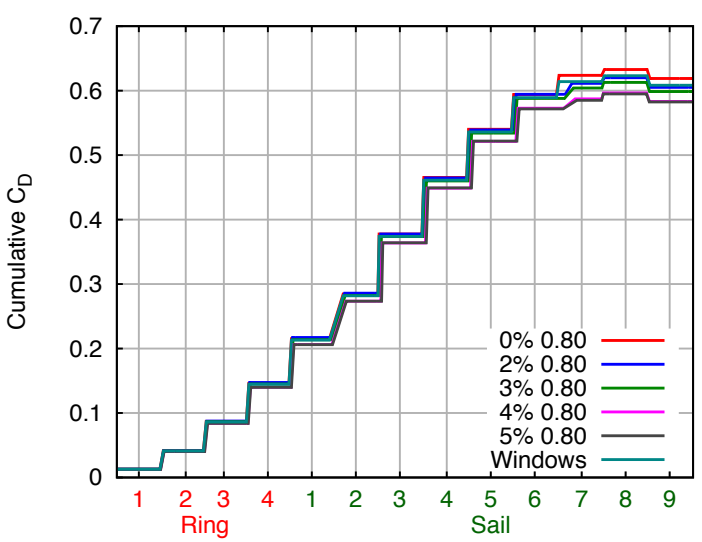

(b) Drag Coefficient

Figure 25. Cumulative normal force and drag coefficients for varying gap sizes at $0.80 h / h_{r}$ with gap at 0.40 $h / h_{r} ; \alpha=20^{\circ}$.

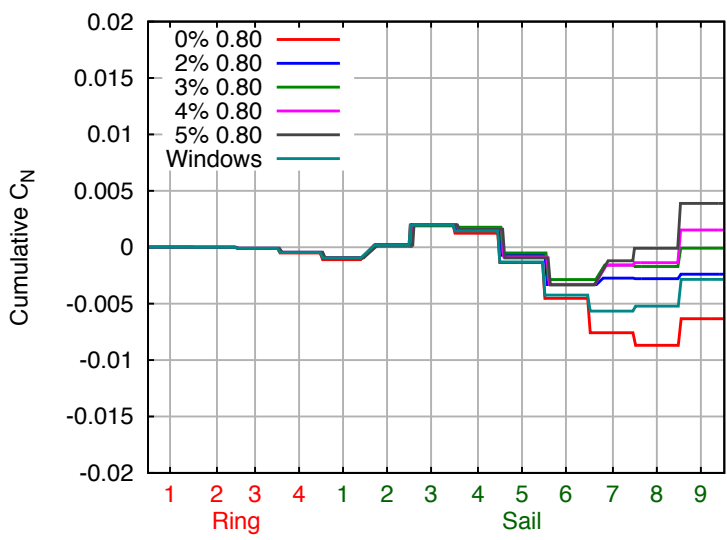

(a) Normal Force Coefficient

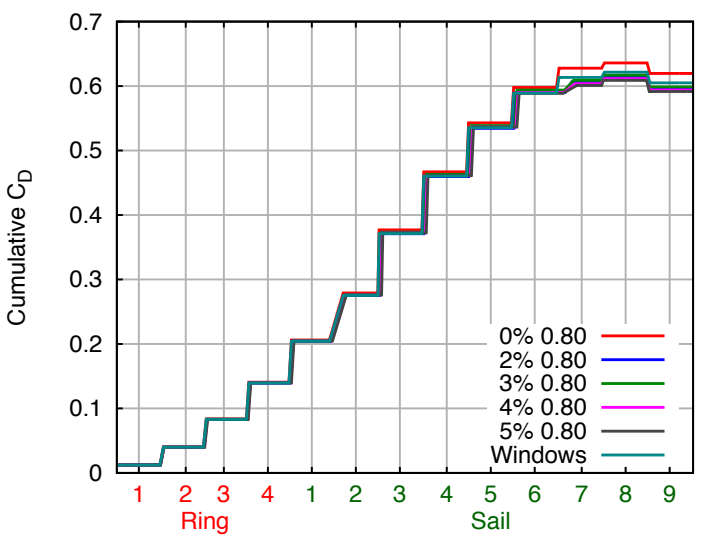

(b) Drag Coefficient

Figure 26. Cumulative normal force and drag coefficients for varying gap sizes at $0.80 h / h_{r}$ with gap at 0.40 $h / h_{r} ; \alpha=\mathbf{1 0}^{\circ}$.

change the $0.40 h / h_{r} 2 \%$ gap configuration due to the unknown changes on initial inflation and 1st and 2nd stage characteristics. If a stability improvement can be found by making changes only to the canopy beyond $0.5 h / h_{r}$, it would allow a large amount of test data to be retained in the certification of the MPCV parachute system. These results indicate that significant stability gains can be obtained by using a combined porosity at both 0.40 and $0.80 h / h_{r}$ while maintaining the flight-tested $2 \%$ gap at $0.40 h / h_{r}$.

\section{E. Super-Sail Geometries}

Another parametric study that was performed focused on the fullness of the sails near the $0.80 h / h_{r}$ location in sail six. Increased porosity at this location on the canopy has already been shown to be beneficial for both static stability and drag. Another means to create porosity at this location is to build sail six with additional fullness. The resulting inflated sail lower edge will be farther from the adjacent sail top edge, resulting in a larger gap and additional geometric porosity. In addition, the additional fullness will result in the inflated sail angle being more closely aligned with the axial direction (centerline of the chute) which may create additional drag relative to a lower fullness sail. When comparing to a canopy having an equivalent geometric porosity created by creating a slot between sails by removing material, the added fullness sail also has the advantage of additional material area to create drag. 
As used in this paper, fullness describes a sail panel's deviation from the theoretical quarter-spherical shape. If constructed to zero fullness, each gore of a sail would have top and bottom widths corresponding to the radius of the quarter sphere at that $h / h_{r}$ height, with the bottom edge naturally being wider than the top edge of the sail. Adding fullness to a canopy involves increasing the width at the bottom of the sail above than the theoretical dimension. It is measured in percent increase from the quarter-sphere measurement. This fullness combined with the tendency of the riser loading to constrict the lower part of the canopy to less than the theoretical spherical shape leads to the crescent shaped gaps between adjacent sails when inflated.

The current MPCV parachute has roughly $10 \%$ fullness at the bottom of sail six. Three additional designs were used in this analysis, each with progressively higher fullness: $25 \%, 35 \%$, and $50 \%$. All parachute shapes include a $3 \%$ porosity slot at $0.40 \%$ to attempt to maximize the stability effect of the $0.40 h / h_{r}$ slot betting that some of the loss in drag will be recovered by use of the higher fullness sail. Unlike the previous analysis, CALA was run to generate the inflated shape of each of the sail six configurations, as there was no intuitive way to create sails of increased fullness within the grid generation tools directly. Sails above approximately $25 \%$ fullness are outside of the experience of the parachute team (25\% max performed for personal chutes). Figure 27 shows an image of the predicted shapes for sail six to show the effect of additional fullness on inflated shape.

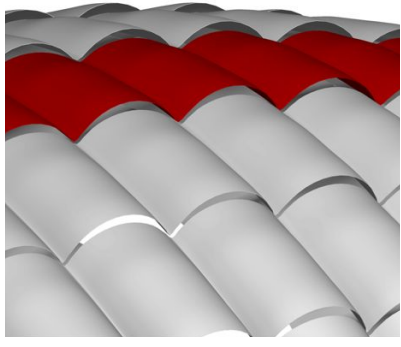

(a) $10 \%$ Fullness

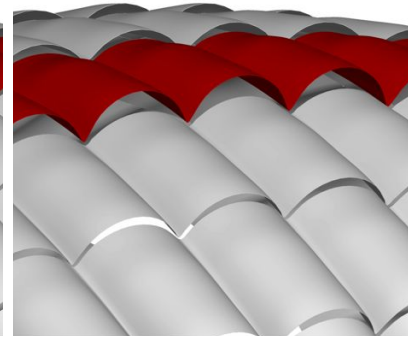

(b) $25 \%$ Fullness

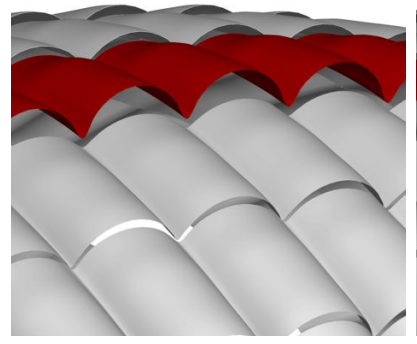

(c) $35 \%$ Fullness

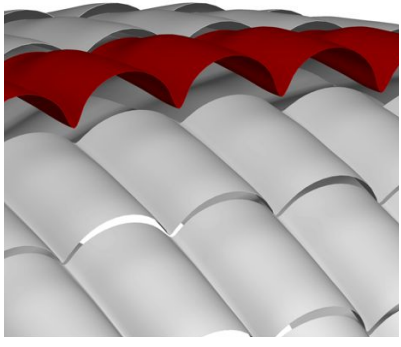

(d) $50 \%$ Fullness

Figure 27. Predicted inflated shape of sail six (red) with varying fullness.

Canopy sails with modified fullness provide a slight increase in porosity. The MPCV EDU currently has $0.4 \%$ geometric porosity between sails six and seven. These modifications increase the porosity to $0.9 \%$, $1.2 \%$, and $1.7 \%$ for the $25 \%, 35 \%$, and $50 \%$ fullness sails, respectively. These represent relatively small increases to porosity compared to the $15 \%$ slots examined in previous studies.

As shown in Fig. 28, even these slight increases result in measurable changes in static stability. While none of the super sail geometries add an additional $2 \%$ porosity, they all predict a statically stable normal force curve. They also do not see as large of a loss in drag performance, and for the $50 \%$ slot, our solutions predict a significant increase in drag by this parachute. These trends are encouraging and suggest that increases in sail fullness can provide an interesting alternative to a uniform slot or gap, or perhaps be used in combination to help offset part of the reduction in drag.

Comparisons of the cumulative loads from vent to skirt show a dramatic effect on the net normal force generated on sail seven. Figure 29 shows the sail and ring contributions at $\alpha=20^{\circ}$. All of the sails with increased fullness see a large rise in cumulative normal force between sails six and seven without a corresponding loss in cumulative drag coefficient. At $\alpha=10^{\circ}$, Fig 30, sails with less than $50 \%$ fullness do not see this large increase in normal force at this location, although slight improvements are evident. Drag performance is also only incrementally improved over the baseline $10 \%$ fullness, and the large improvement shown by the $50 \%$ fullness sail at $\alpha=10^{\circ}$ is not evident here.

Figures 31 and 32 show the contribution of each panel to the overall normal force of each canopy for varying fullness at $\alpha=20^{\circ}$ and $\alpha=10^{\circ}$. There are some consistent trends for both values of $\alpha$. When adding fullness, the windward portion of sail six (bottom of figures) has forward facing porosity interact with shear layer and 'stagnate' flow on sails seven to nine. This increases the pressure on the outside of the parachute and reduces the pressure delta inside and out. On the leeward side (top), it creates attached flow on sail seven that drives an even higher pressure differential inside-to-outside. This increases the normal force contribution from sail seven. Both effects contribute to the improved static stability. 


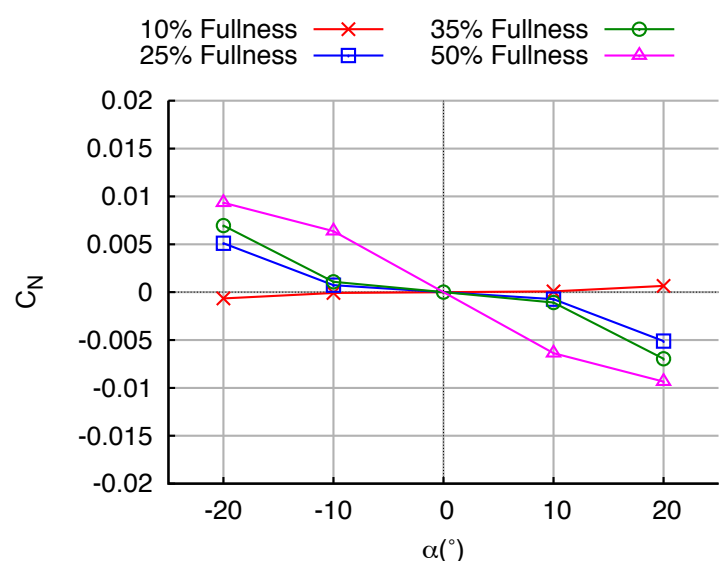

(a) Normal Force Coefficient

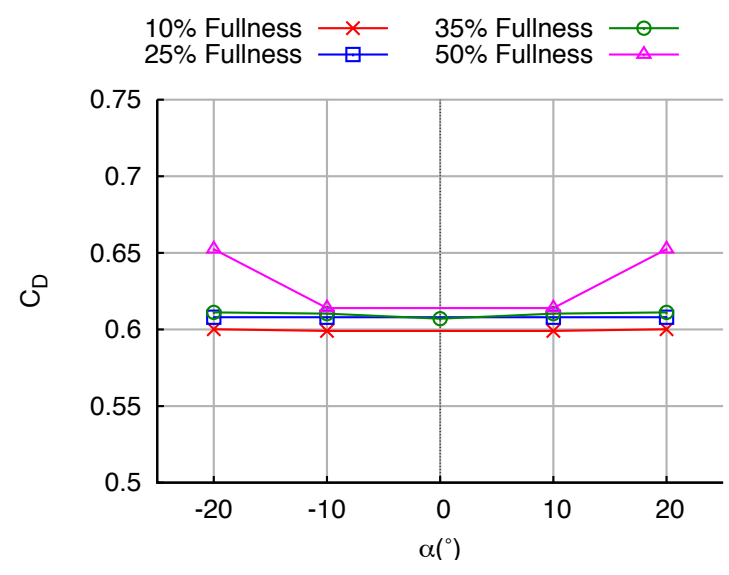

(b) Drag Coefficient

Figure 28. Integrated drag and normal force coefficients for varying fullness in sail six.

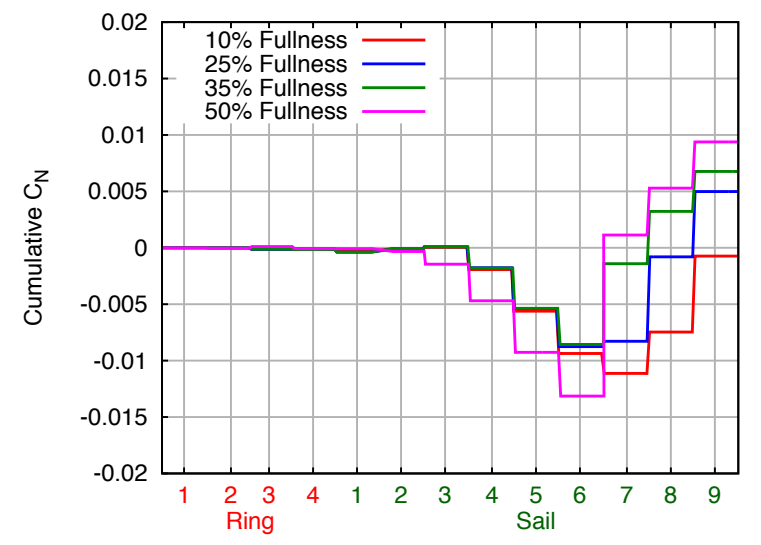

(a) Normal Force Coefficient

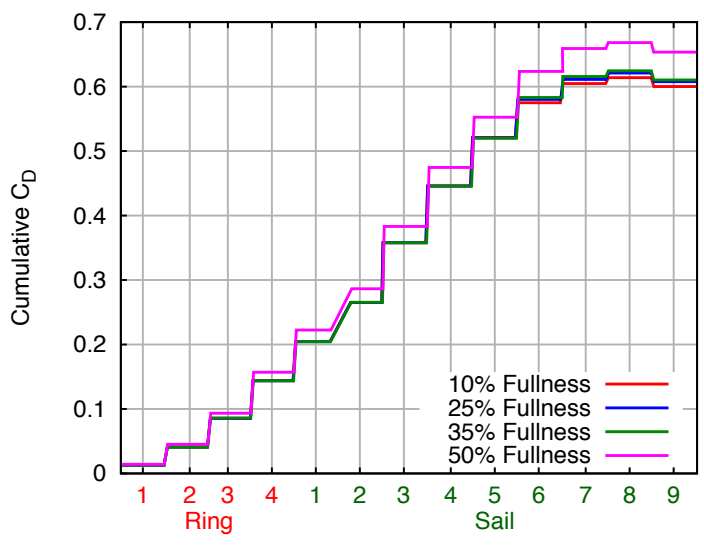

(b) Drag Coefficient

Figure 29. Cumulative normal force and drag coefficients for varying sail 6 fullness; $\alpha=20^{\circ}$.

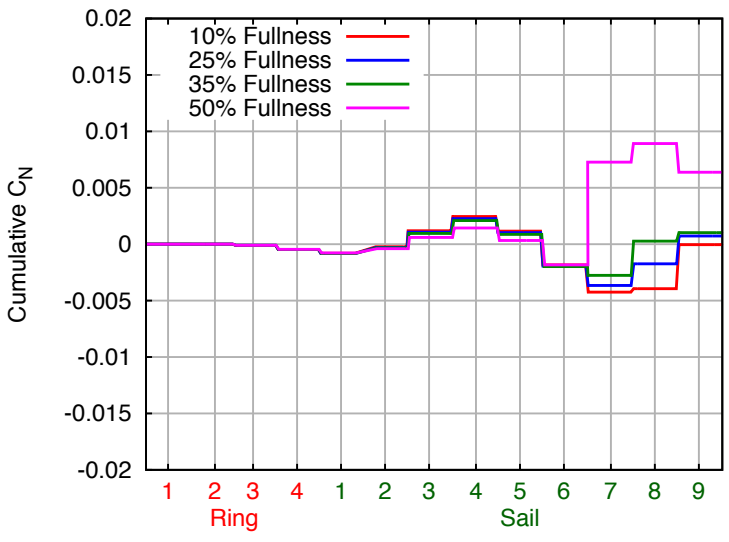

(a) Normal Force Coefficient

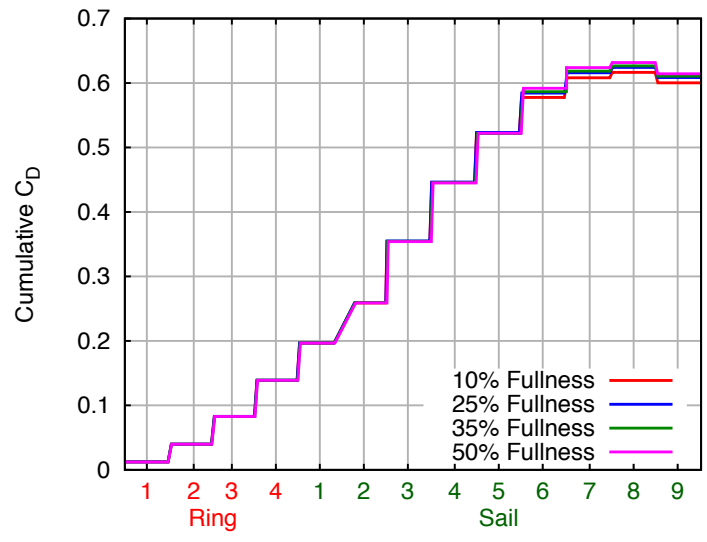

(b) Drag Coefficient

Figure 30. Cumulative normal force and drag coefficients for varying sail 6 fullness; $\alpha=10^{\circ}$. 


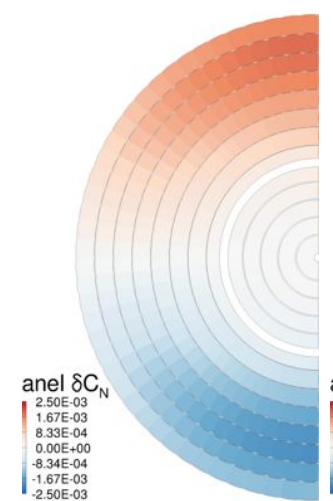

(a) $10 \%$ Fullness

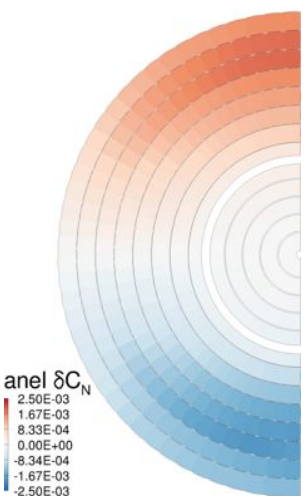

(b) $25 \%$ Fullness

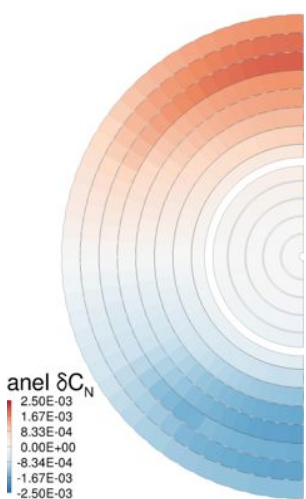

(c) $35 \%$ Fullness

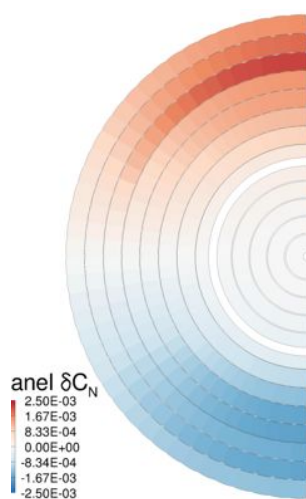

(d) $50 \%$ Fullness

Figure 31. Panel contributions to normal force coefficient for canopies with varying fullness in sail six; $\alpha=20^{\circ}$.

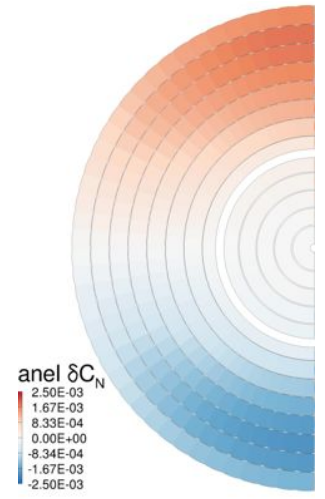

(a) $10 \%$ Fullness

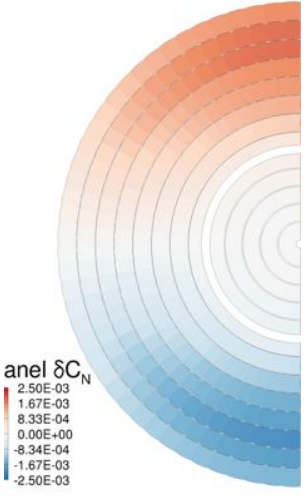

(b) $25 \%$ Fullness

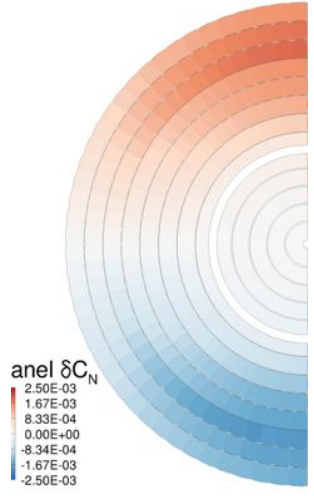

(c) $35 \%$ Fullness

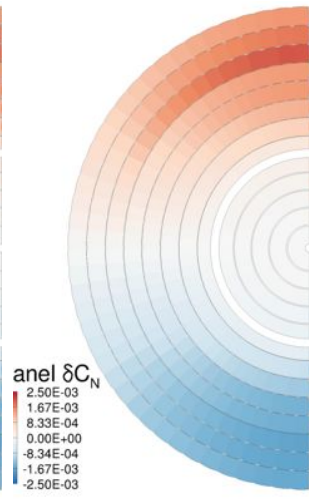

(d) $50 \%$ Fullness

Figure 32. Panel contributions to normal force coefficient for canopies with varying fullness in sail six; $\alpha=10^{\circ}$.

\section{Conclusions and Further Work}

This work presents a technique for rapidly generating computational assets to analyze parachute decelerators using computational fluid dynamics. This technique combines the CALA structural solver for geometry definition, the CGT package for precise discretization of the resulting inflated shapes, and the Navier-Stokes solver OVERFLOW to obtain flowfield and integrated aerodynamics. The flexibility of these methods allow engineers a new capability for parameter analysis and experimentation that is relatively inexpensive.

Validation cases designed to mimic drop test data from NASA's Apollo program are shown. The trends between the computational and experimental data show promising results. Even though this method does not attempt to capture the dynamic effects from the moving parachute canopy, it shows qualitative agreement.

The analysis performed here predicts that the existing MPCV parachute is statically unstable. This is hypothesized to be a contributor to the pendulum phenomenon. Subsequent studies of geometric porosity explore possible modifications to effect positive change in static stability while minimizing impact to canopy drag.

Adding a large ring of porosity at a gore height of $0.40 h / h_{r}$ showed significant improvement for static stability near $\alpha=10^{\circ}$, which is consistent with the Apollo parachute results. Parachute stability vs. porosity percentage showed a pronounced non-linearity at this $h / h_{r}$ location with a significant change around $3 \%$ porosity. Additional porosity beyond this point did not yield an increase in parachute static stability, but did incur a drag penalty. It should be noted that modifications to the MPCV parachute at this location will have consequences for inflation dynamics and reefed performance not explored in this work.

Rings of porosity at $0.80 h / h_{r}$ show an ability to increase parachute normal force most effectively at 
$\alpha=20^{\circ}$. The trends are generally linear and increases to porosity at this location do not show a pronounced reduction in drag coefficient, when the likely effects on inflated diameter are suppressed.

Parachutes with rings of porosity at both $0.40 h / h_{r}$ and $0.80 h / h_{r}$ have improved static stability for the entire range of $\alpha$ explored here. Drag loss is similarly reduced, and canopy performance appears to be slightly better than superposition would predict. Results indicate that discrete points of porosity (missing panels) do not improve static stability as well as a concentric ring of porosity of similar geometric area, but does have a slightly lower drag penalty.

The concept of the 'super sail' with dramatically increased fullness provides an alternative to a constant gap and adds geometric porosity. Canopies with this modification to sail six do not appear to suffer as pronounced of a drag loss as a concentric ring of porosity and offer similar benefits. Not shown here are promising results that combine increased fullness with a concentric gap. A ring-sail parachute of this size with fullness of the magnitude examined here is unprecedented and future work with experimental methods are important to ensure that additional fullness does not compromise inflated shape.

This work has examined static stability only. Parachute aerodynamics have significant contributions from dynamics and these data were run in a static configuration. OVERFLOW can run moving-body simulations with prescribed-motion as well as constrained simulations with a canopy allowed to freely pivot due to the aerodynamics. Future work on this problem will incorporate improvements to the grid scripts so that this capably can be leveraged for this problem. An ability to compute dynamic stability terms would greatly improve the functionality of this tool.

Forward work the authors feel need to be undertaken, first involves additional validation vs more quantitive data than the Apollo system oscillation angle data used.

Finally, there are a number of avenues that are currently or will be explored to improve the fidelity of this analysis. They include: additional validation of these tools vs more quantitate data than the Apollo system oscillation results used to date, use of detached-eddy simulation to reinforce or refute the conclusions mentioned here by modeling a more rich range of turbulent content, improvements to the flow solver to enable modeling of material permeability based on the pressure across the canopy, specified rotation or 1DOF time-accurate motion to assess both pitch damping and perhaps flow hysteresis, and loosely coupling a structural solver with the flow solver to allow fluid-structure interaction that would modify the parachute shape based on predicted canopy pressures.

In support of MPCV parachute design, sub-scale wind tunnel and flight tests have been executed with considerable instrumentation on the parachute canopies and drop test payloads. These test incorporate some of the modifications presented here and the data collected present a unique opportunity to better understand this technique and improve it for use in future decelerator system design.

\section{Acknowledgements}

The authors would like to acknowledge the contributions from the MPCV Pendulum Action Team for their insight and assistance throughout this effort. The team consists of engineers across NASA, Lockheed Martin, Jacobs Engineering, and Airborne Systems. Due to the long list of participants, and fear of omitting anyone, we recognize them as a group. Their advice and judgment should be considered instrumental to this effort.

\section{References}

${ }^{1}$ E. S. Ray and R. A. Machin, "Pendulum Motion in Main Parachute Clusters," in 23rd AIAA Aerodynamic Decelerator Systems Technology Conference, Submitted, 2015.

${ }^{2}$ W. D. Sundberg, "A New Solution Method for Steady-State Canopy Structural Loads," No. AIAA 86-2489, 1986.

${ }^{3}$ S. E. Rogers, K. Roth, M. Field, S. M. Nash, M. D. Baker, J. P. Slotnick, M. Whitlock, L. Beach, and H. V. Cao, "Advances in Overset CFD Processes Applied to Subsonic High-Lift Aircraft," No. AIAA 2000-4216, 2000.

${ }^{4}$ W. M. Chan, "The Overgrid Interface for Computational Simulations on Overset Grids," No. AIAA 2002-3188, 2002.

${ }^{5}$ S. E. Rogers, U. S. Army, and R. Arsenal, "PEGASUS 5 : An Automated Preprocessor for Overset-Grid Computational Fluid Dynamics Introduction," vol. 41, no. 6, pp. 1037-1045, 2003.

${ }^{6}$ R. H. Nichols, R. W. Tramel, D. Fusion, P. G. Buning, S. A. Engineer, A. Engineer, and C. A. Branch, "Solver and Turbulence Model Upgrades to OVERFLOW 2 for Unsteady and High-Speed Applications," No. AIAA 2006-2824, 2006.

${ }^{7}$ S. Belknap and K. Goar, "Summary Analysis Report of Drop Tests Performed During the Apollo Main Parachuge Improvement Program," Tech. Rep. NVR-3722, 1964. 
${ }^{8}$ S. S. Nash-Boulden and D. C. Coe, "Analysis of the Full Scale and Clustered One-Third Scale Apollo 88 Foot Ringsail Parachute Tests in the Ames 40x80 Foot Wind Tunnel - Second Series," Tech. Rep. NVR-3518, 1964. 